Supplement of The Cryosphere Discuss., 8, 4463-4495, 2014

http://www.the-cryosphere-discuss.net/8/4463/2014/

doi:10.5194/tcd-8-4463-2014-supplement

(C) Author(s) 2014. CC Attribution 3.0 License.

(c) (1)
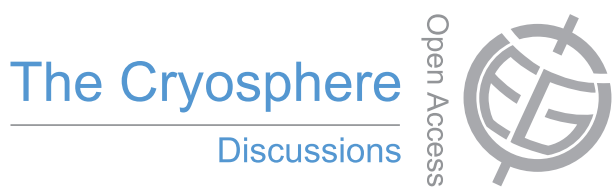

Supplement of

\title{
A conceptual model of cyclical glacier flow in overdeepenings
}

\section{J. B. Turrin and R. R. Forster}

Correspondence to: J. B. Turrin (jturrin@ hotmail.com) 


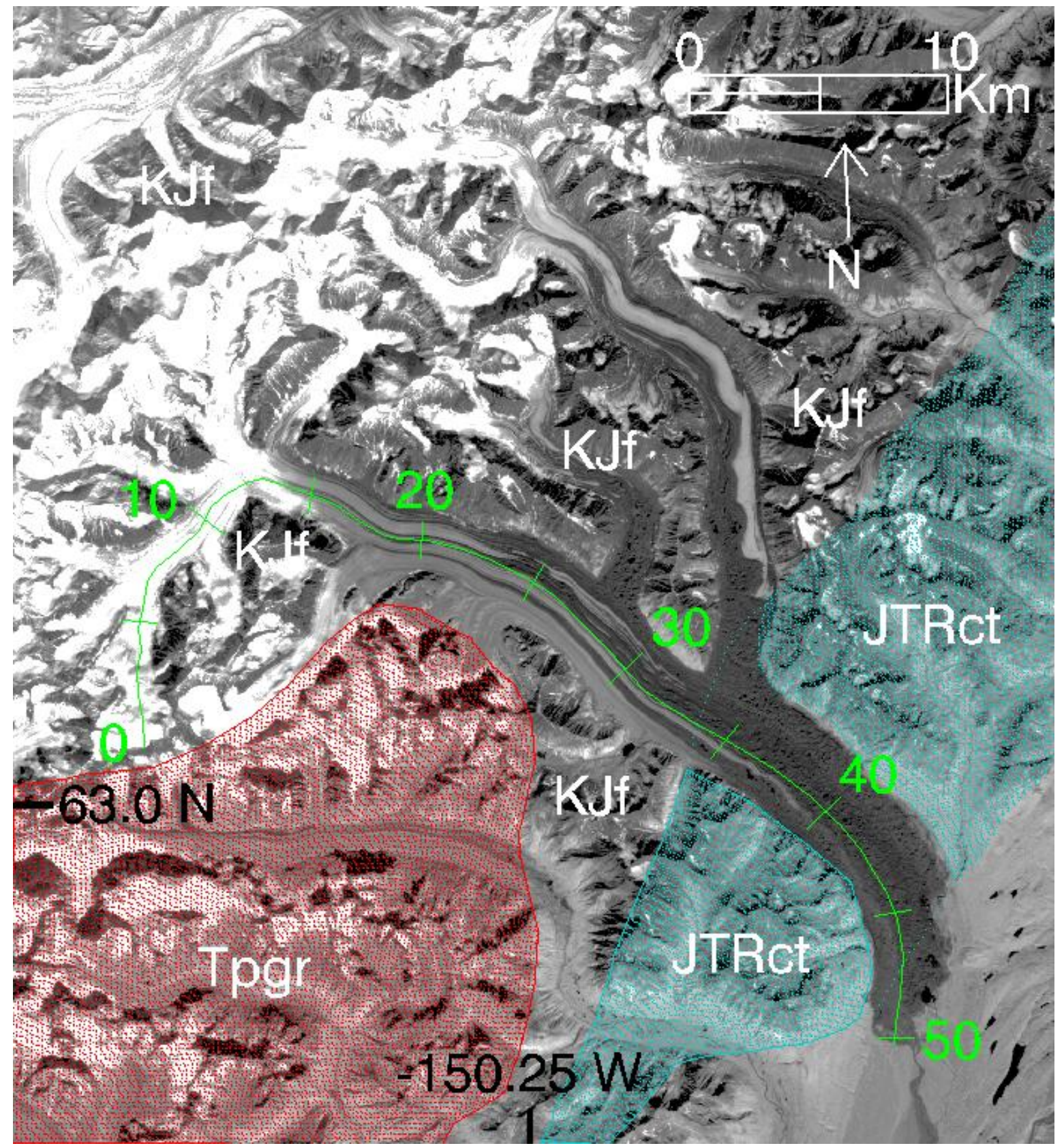

Figure S1. Eldridge Glacier. Transect along glacier centerline (shown in green) indicates the location of the velocity profiles shown in Figure S9, units are km. Areas highlighted in aqua and labeled JTRct indicate the location of bedrock composed primarily of crystalline tuff. Areas highlighted in red and labeled Tpgr indicate the location of bedrock composed of granite. Areas labeled KJf are underlain by sedimentary bedrock (Wilson et al., 1998). 

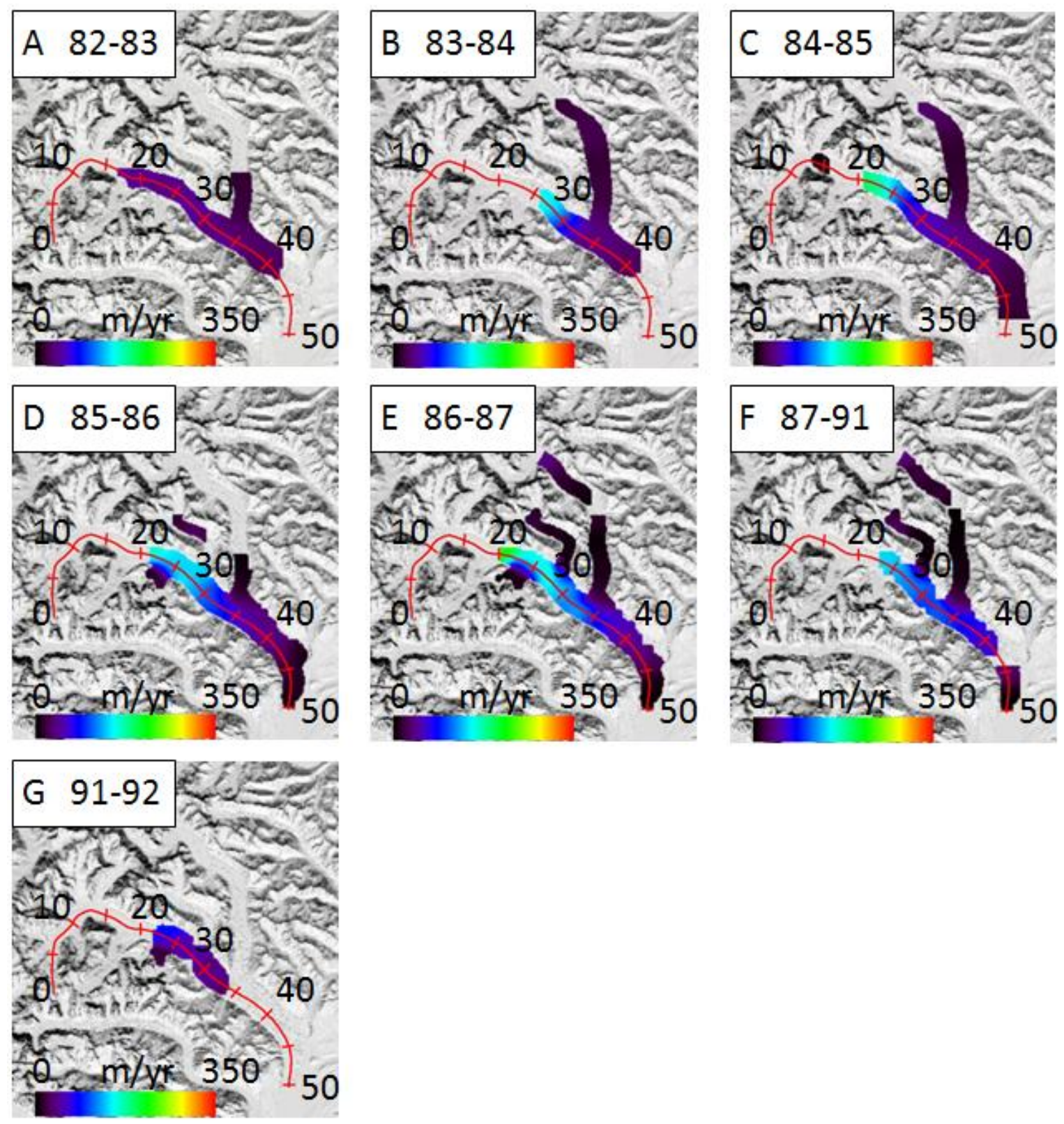

Figure S2. Velocity fields for Eldridge Glacier encompassing the first pulse from 1982-83 to 1991-92. A) 1982-83 velocity. B) 1983-84 velocity. C) 1984-85 velocity. D) 1985-86 velocity. E) 1986-87 velocity. F) 1987-91 velocity. G) 1991-92 velocity. Velocity fields for the second pulse of Eldridge Glacier are not shown. 

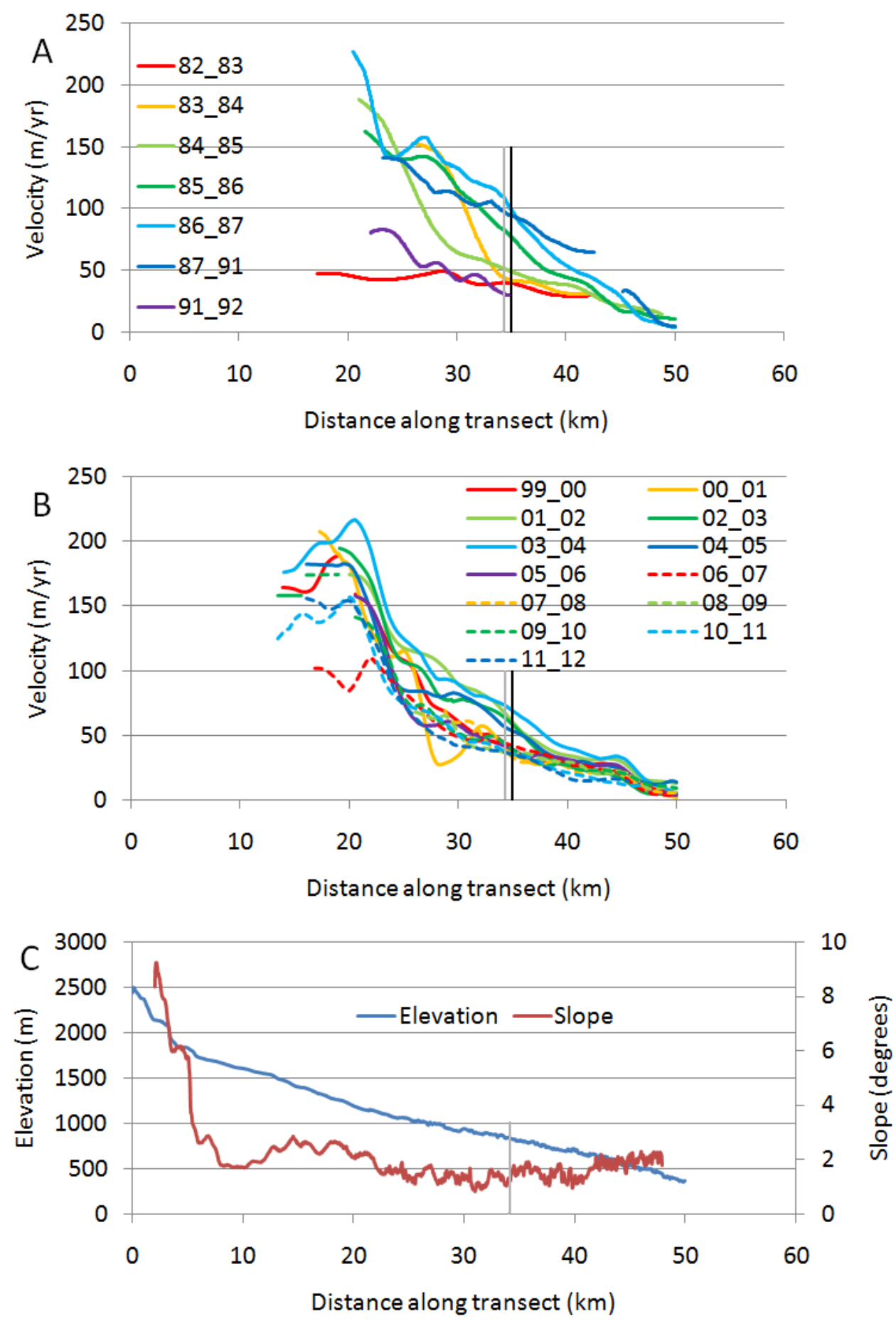

Figure S3. Centerline profiles for Eldridge Glacier. A) Velocity from 1982-83 to 1991-92 showing the first pulse. B) Velocity from 1999-00 to 2011-12 showing the second pulse. C). Elevation and slope of the glacier surface. Vertical black line at $35 \mathrm{~km}$ in panels A and B indicates position at which velocity values were taken to create time-series in Fig. S8. Vertical gray line at $34 \mathrm{~km}$ indicates location of major change in bedrock lithology. 


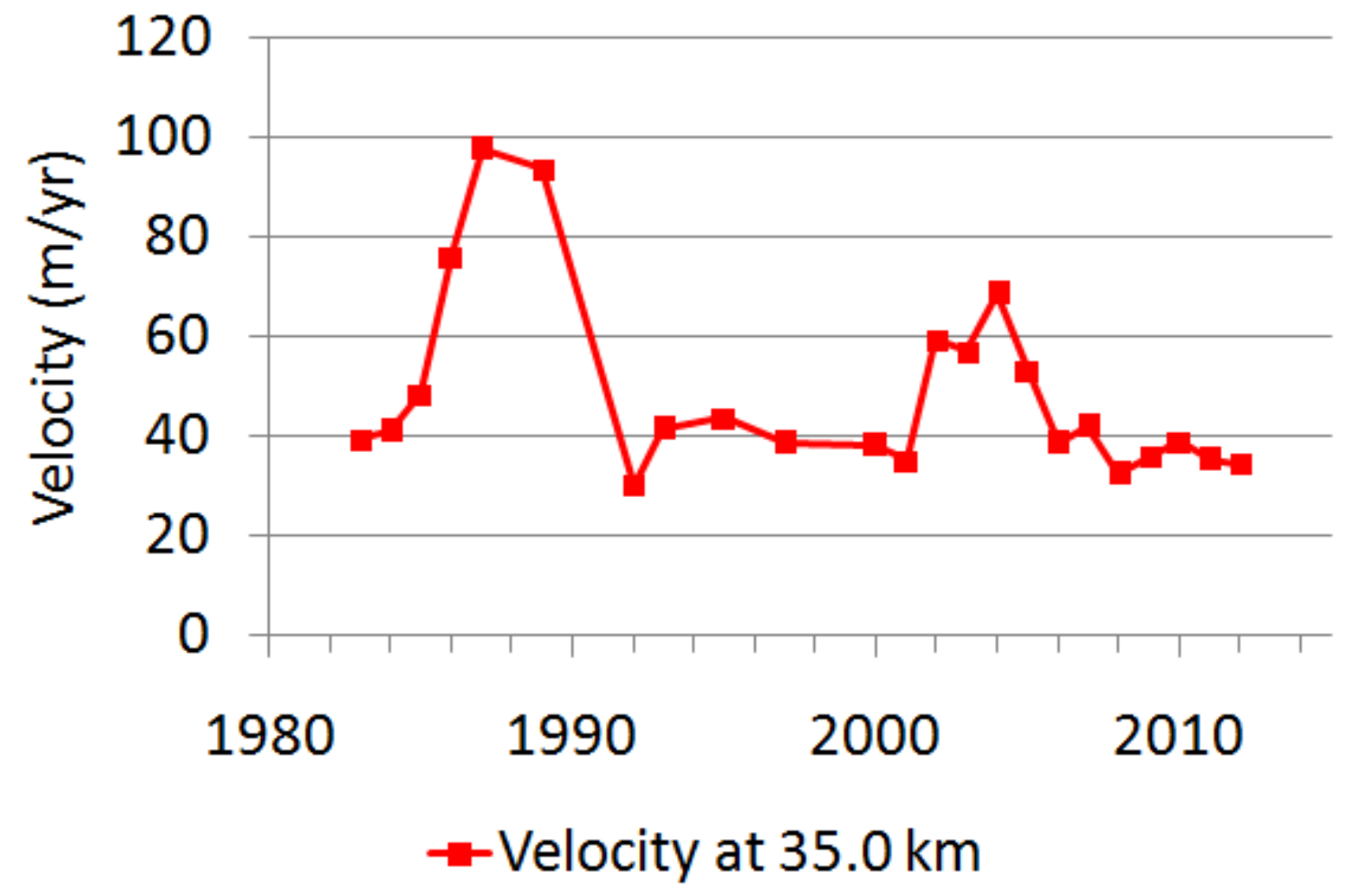

Figure S4. Velocity of Eldridge Glacier through time, at $35 \mathrm{~km}$. Two pulses are seen; the first pulse peaked in 1987 at $97.8 \mathrm{~m} / \mathrm{yr}$, and the second pulse peaked in 2004 at $68.8 \mathrm{~m} / \mathrm{yr}$. 


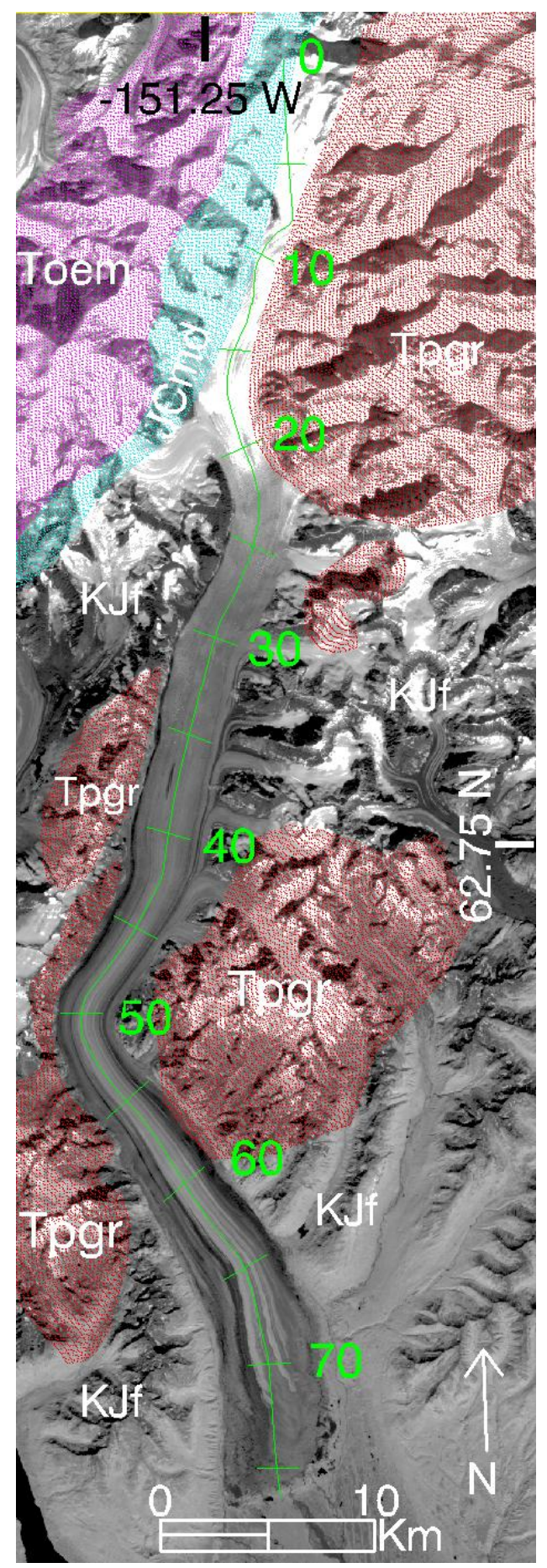

Figure S5. Kahiltna Glacier. Transect along glacier centerline (shown in green) indicates the location of the velocity profiles shown in Figure S7, units are $\mathrm{km}$. Areas highlighted in red (Tpgr) and magenta (Toem) indicate areas underlain by crystalline bedrock composed of granite or granodiorite, respectively. Areas highlighted in aqua (JCmd) or labeled KJf indicate areas underlain by sedimentary bedrock (Wilson et al., 1998). 

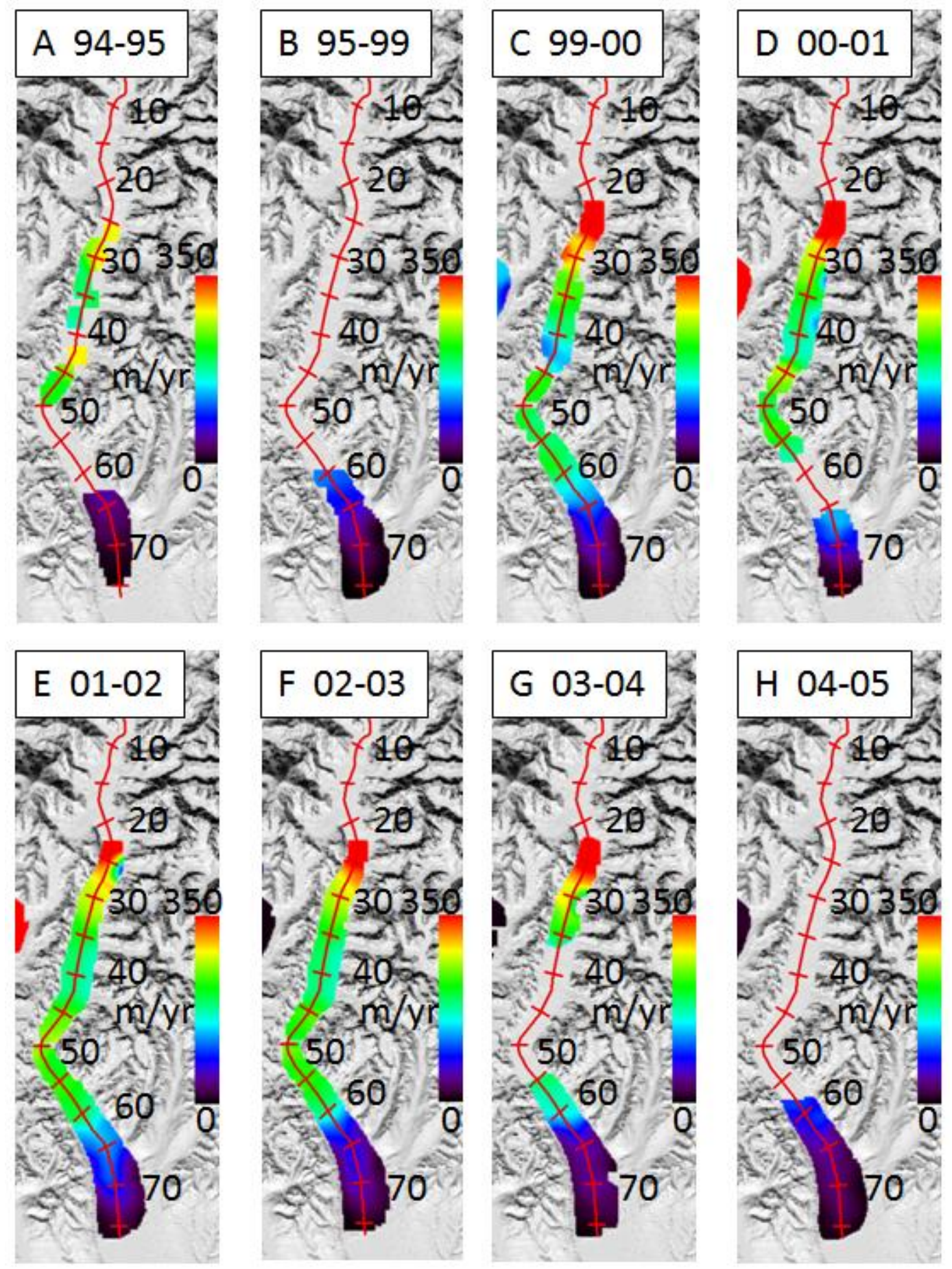

Figure S6. Velocity fields for Kahiltna Glacier encompassing the first pulse from 1994-95 to 2004-05. A) 1994-95 velocity. B) 1995-99 velocity. C) 1999-00 velocity. D) 2000-01 velocity. E) 2001-02 velocity. F) 2002-03 velocity. G) 2003-04 velocity. H) 2004-05 velocity. Velocity fields for the second pulse of Kahiltna Glacier are not shown. 

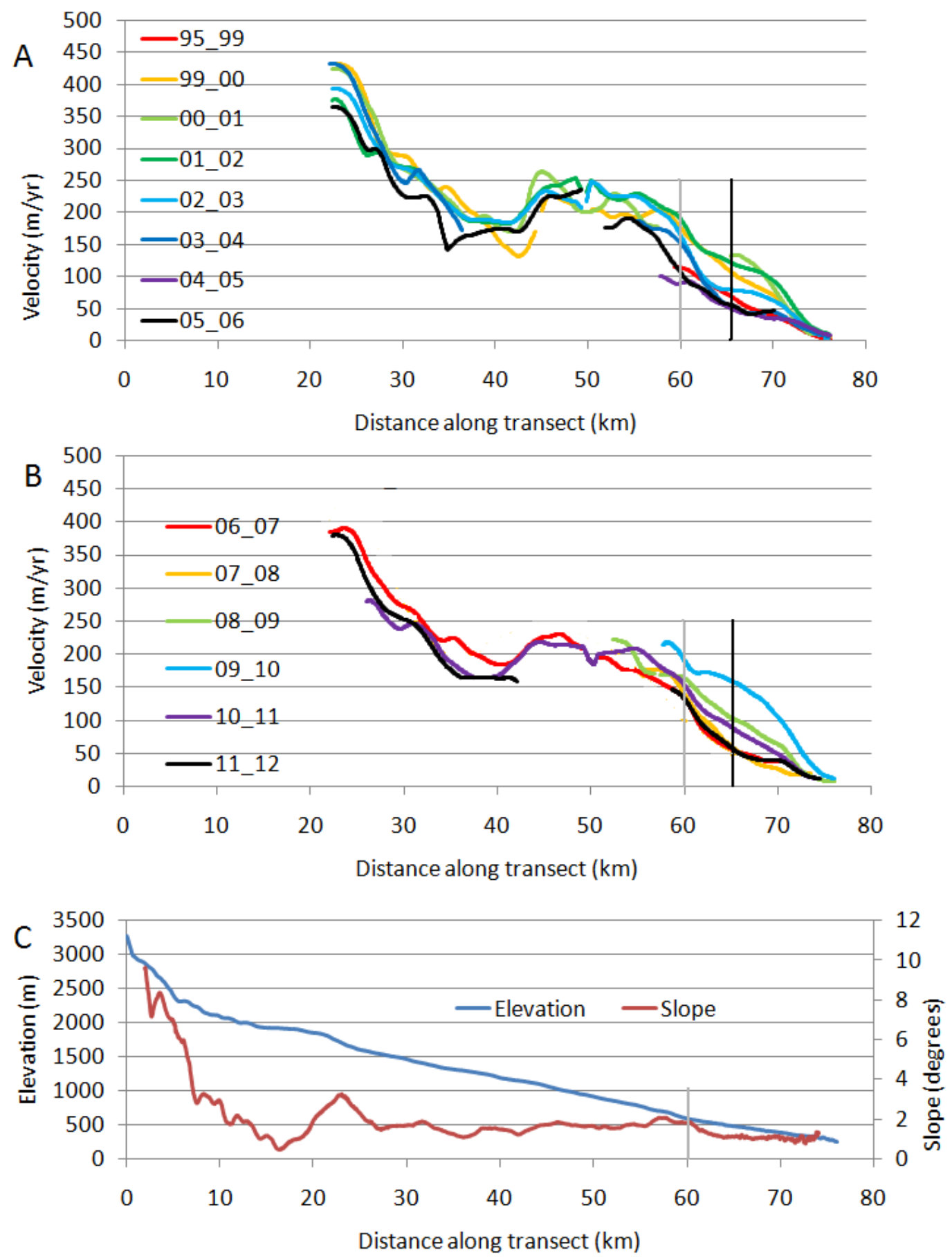

Figure S7. Centerline profiles for Kahiltna Glacier. A) Velocity from 1995-99 to 2005-06 showing the first pulse. B) Velocity from 2006-07 to 2011-12 showing the second pulse. Dashed orange profile represents velocity measurements in 2007/08 by Goodwin et al. (2012). C). Elevation and slope of the glacier surface. Vertical black line at $65 \mathrm{~km}$ in panels A and B indicates position at which velocity values were taken to create time-series in Fig. S8. Vertical gray line at $60 \mathrm{~km}$ indicates location of major change in bedrock lithology. 


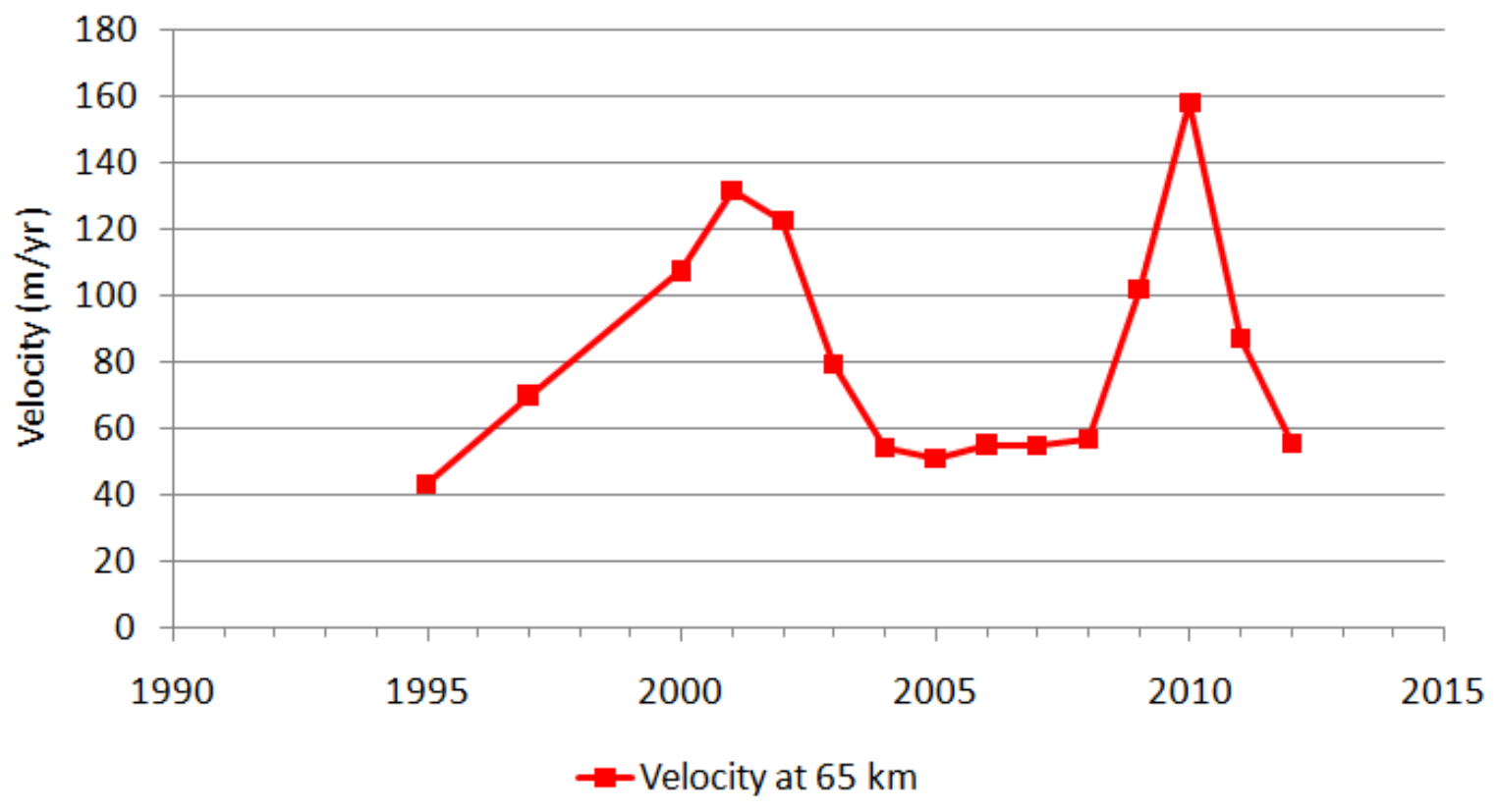

Figure S8. Velocity of Kahiltna Glacier through time, at $65 \mathrm{~km}$. Two pulses are seen; the first pulse peaked in 2001 at $131.4 \mathrm{~m} / \mathrm{yr}$, and the second pulse peaked in 2010 at $157.5 \mathrm{~m} / \mathrm{yr}$. 


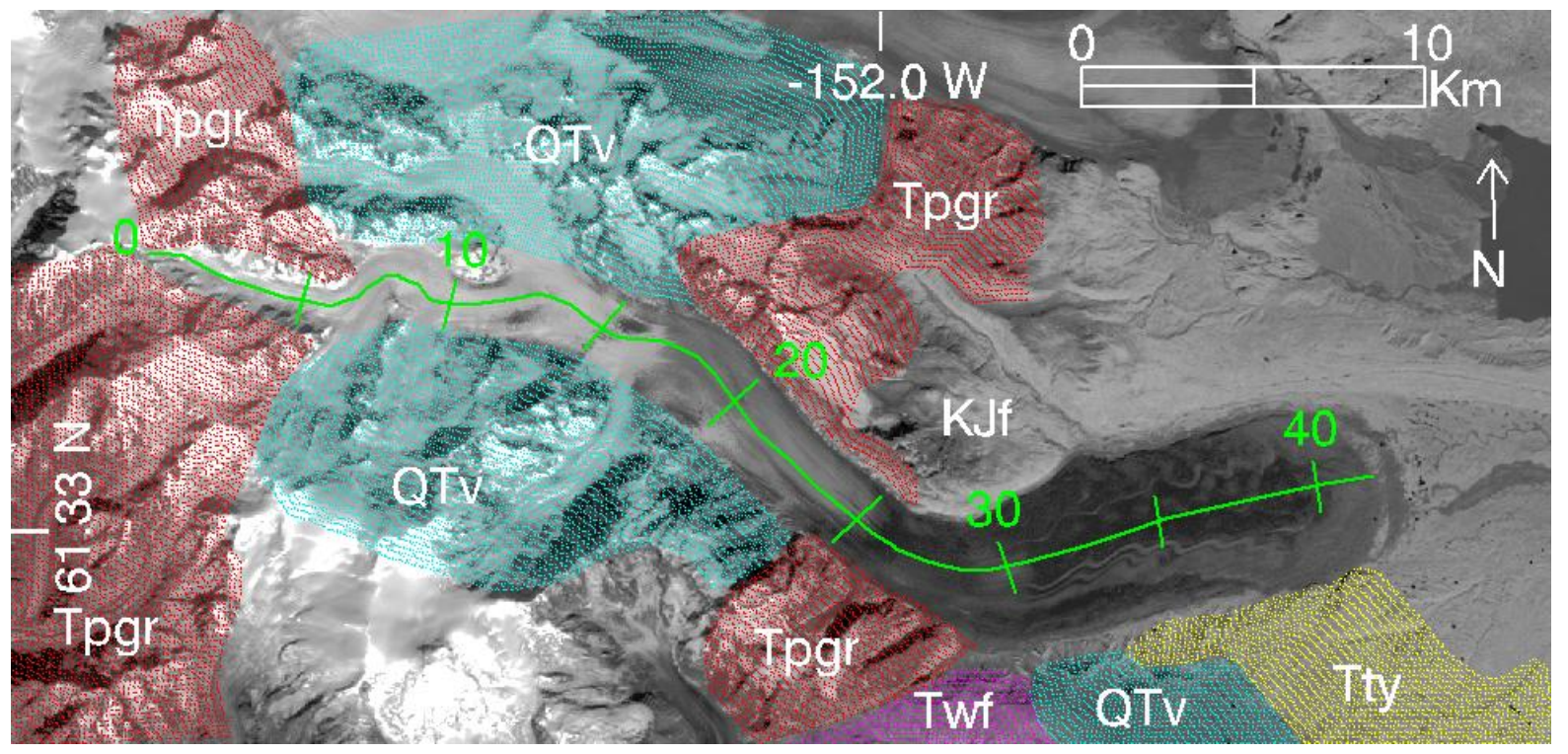

Figure S9. Capps Glacier. Transect along glacier centerline (shown in green) indicates the location of the velocity profiles shown in Figure S11, units are km. Areas highlighted in red or aqua indicate areas underlain by crystalline bedrock composed of granite (Tpgr) or basalt (QTv), respectively. Areas highlighted in magenta (Twf) or yellow (Tty), or labeled KJf, indicate areas underlain by sedimentary bedrock (Wilson et al., 1998). 

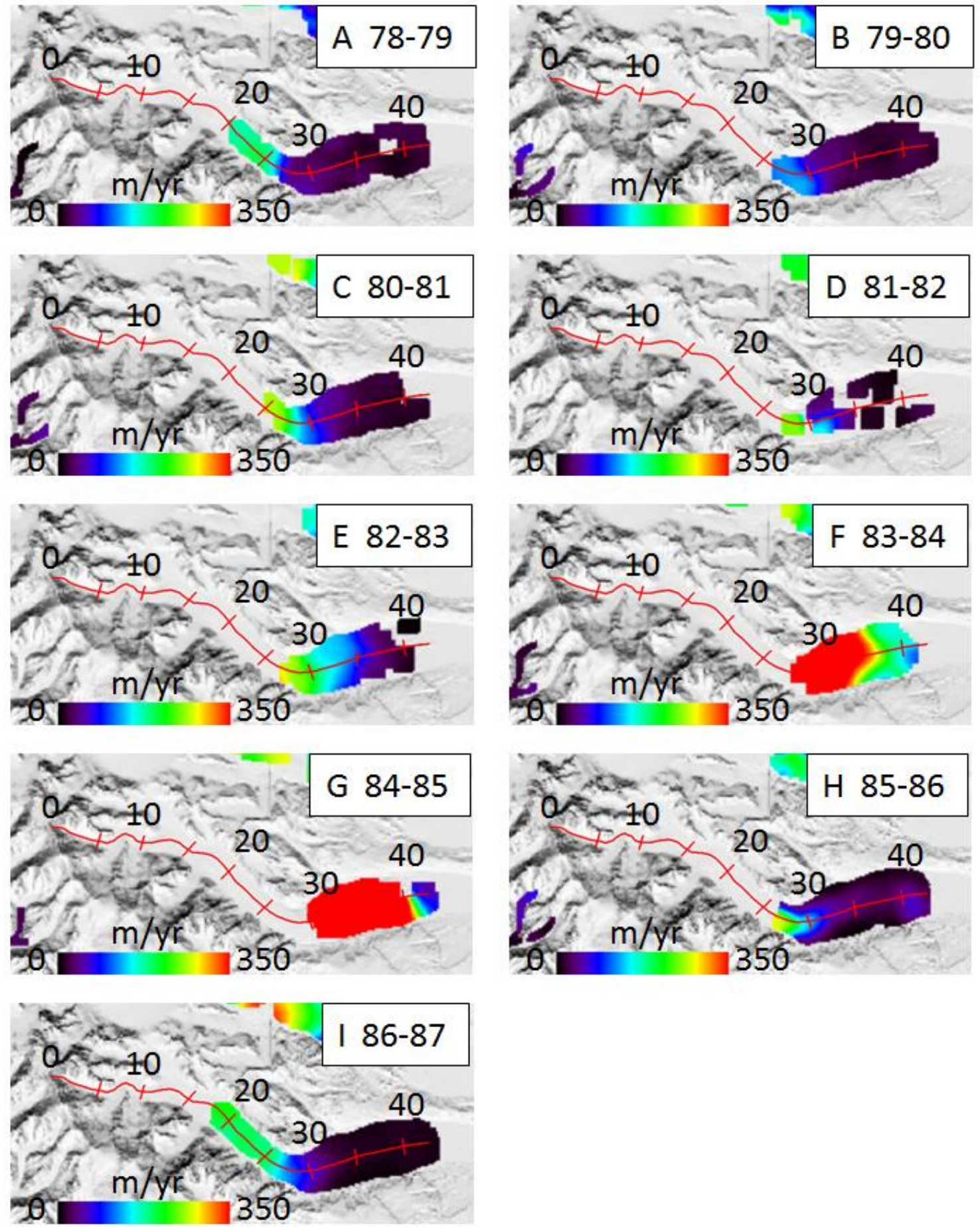

Figure S10. Velocity fields for Capps Glacier encompassing the second pulse from 1978-79 to 1986-87. A) 1978-79 velocity. B) 1979-80 velocity. C) 1980-81 velocity. D) 1981-82 velocity. E) 1982-83 velocity. F) 1983-84 velocity. G) 1984-85 velocity. H) 1985-86 velocity. I) 198687 velocity. Velocity fields for the first and third pulses of Capps Glacier are not shown. 

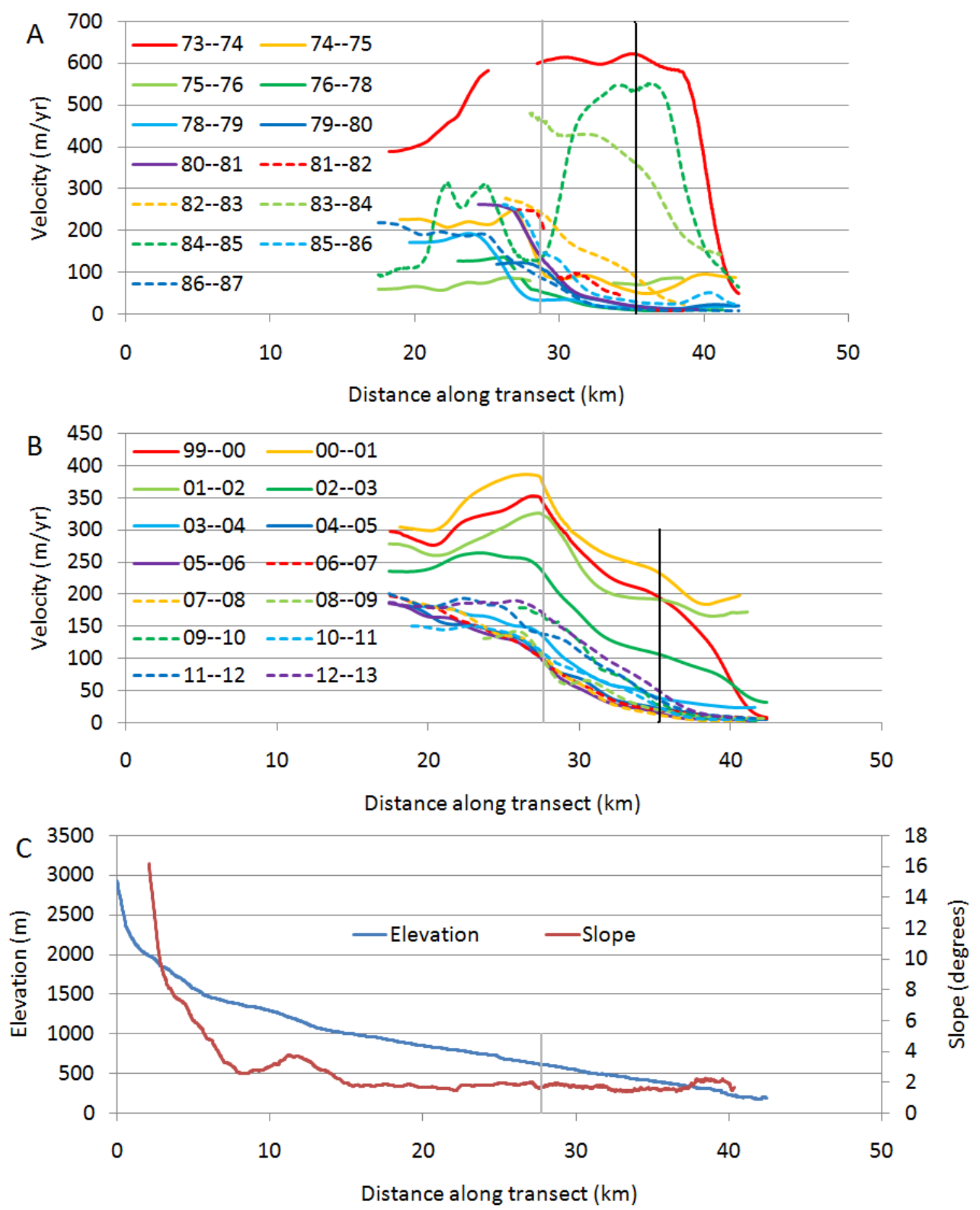

Figure S11. Centerline profiles for Capps Glacier. A) Velocity profiles from 1973-74 to 198687 showing the first and second pulses. B) Velocity profiles from 1999-00 to 2012-13 showing the third pulse. C) Elevation and slope of the glacier surface. Vertical black line at $35 \mathrm{~km}$ in panels $\mathrm{A}$ and $\mathrm{B}$ indicates position at which velocity values were taken to create time-series in Fig. S12. Vertical gray line at $28 \mathrm{~km}$ indicates location of major change in bedrock lithology. 


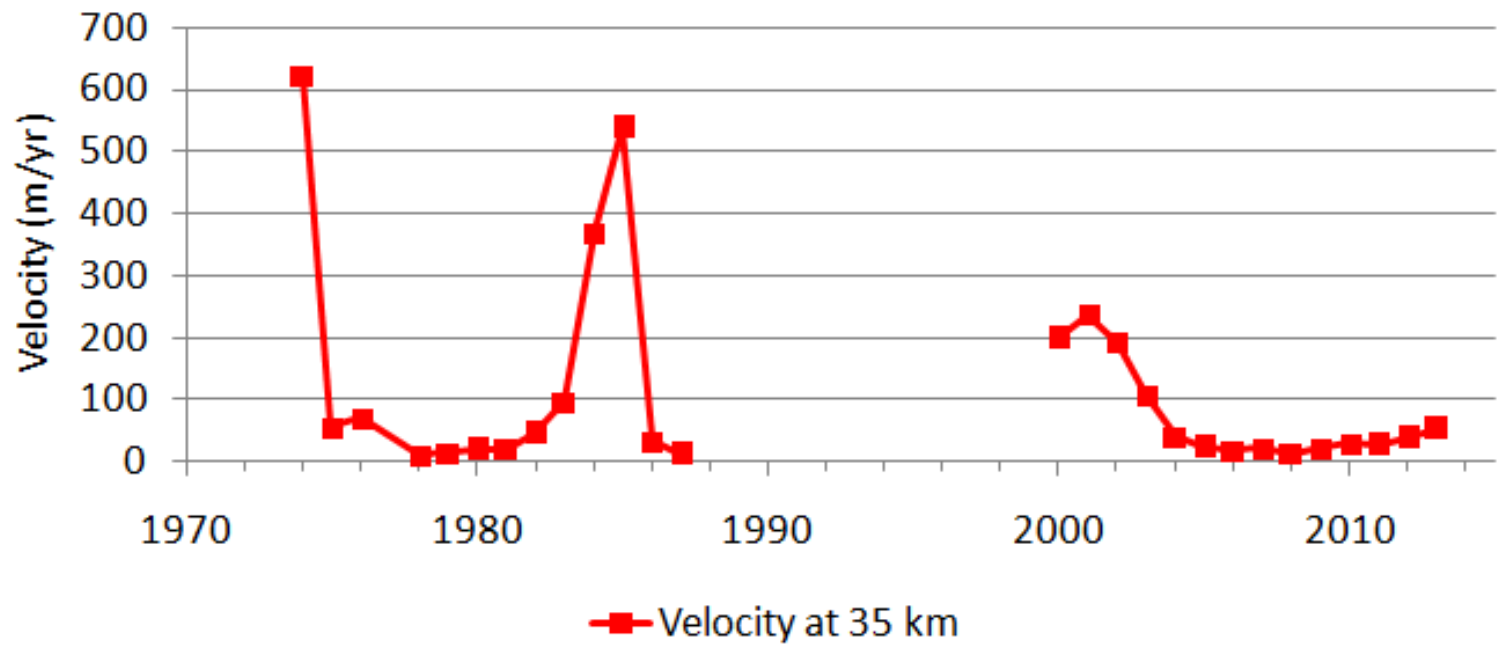

Figure S12. Velocity of Capps Glacier through time, at $35 \mathrm{~km}$. Three pulses are seen; the first pulse peaked in 1974 at $632.2 \mathrm{~m} / \mathrm{yr}$, the second pulse peaked in 1985 at $537.9 \mathrm{~m} / \mathrm{yr}$, and the third pulse peaked in 2001 at $237.2 \mathrm{~m} / \mathrm{yr}$. 


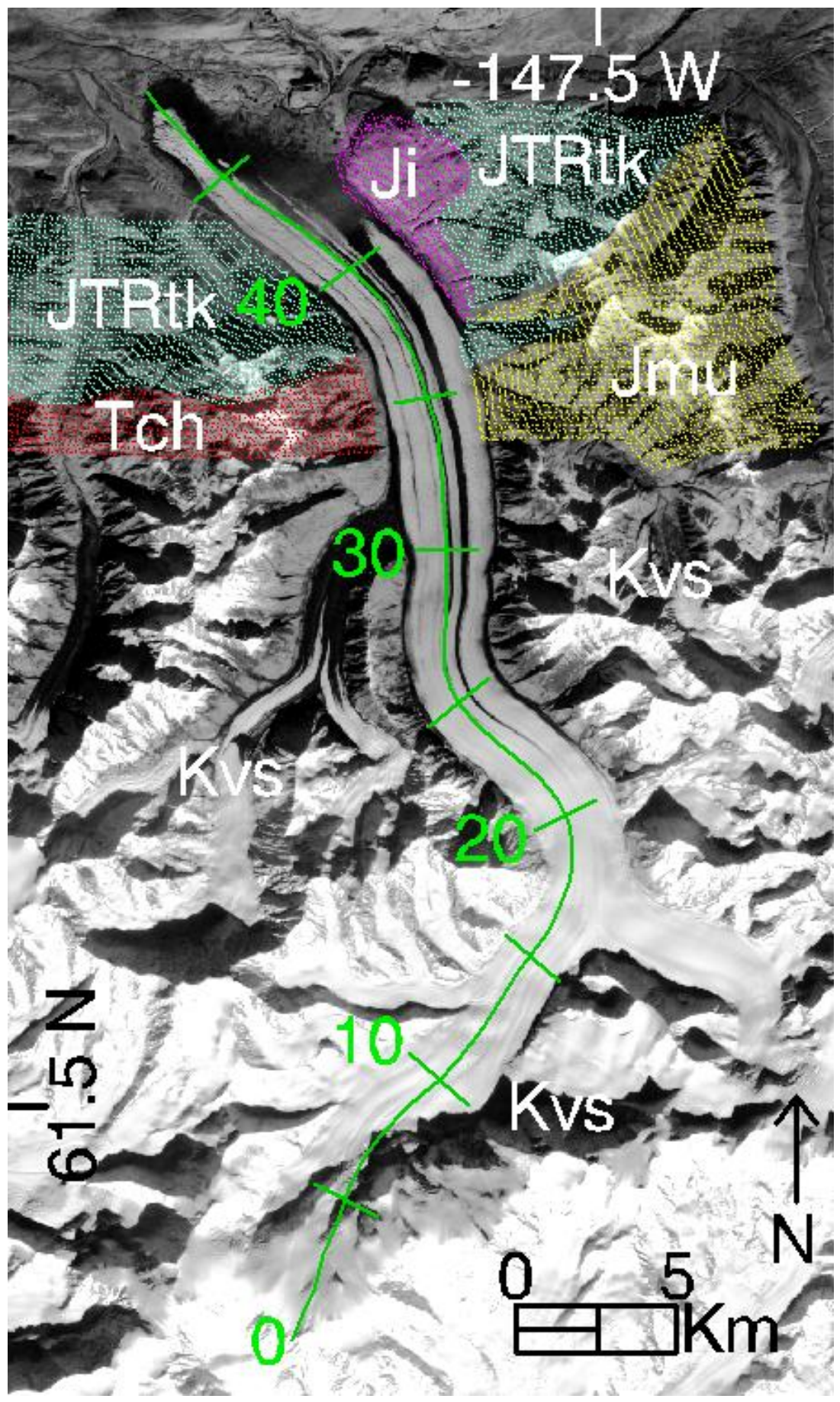

Figure S13. Matanuska Glacier. Transect along glacier centerline (shown in green) indicates the location of the velocity profiles shown in Figure S15, units are km. Areas highlighted in magenta (Ji) or yellow (Jmu) indicate areas underlain by crystalline bedrock composed of granite or various igneous ultramafic rocks, respectively. Areas highlighted in aqua (JTRtk) or red (Tch), or labeled Kvs, indicate areas underlain by sedimentary bedrock (Wilson et al., 1998). 

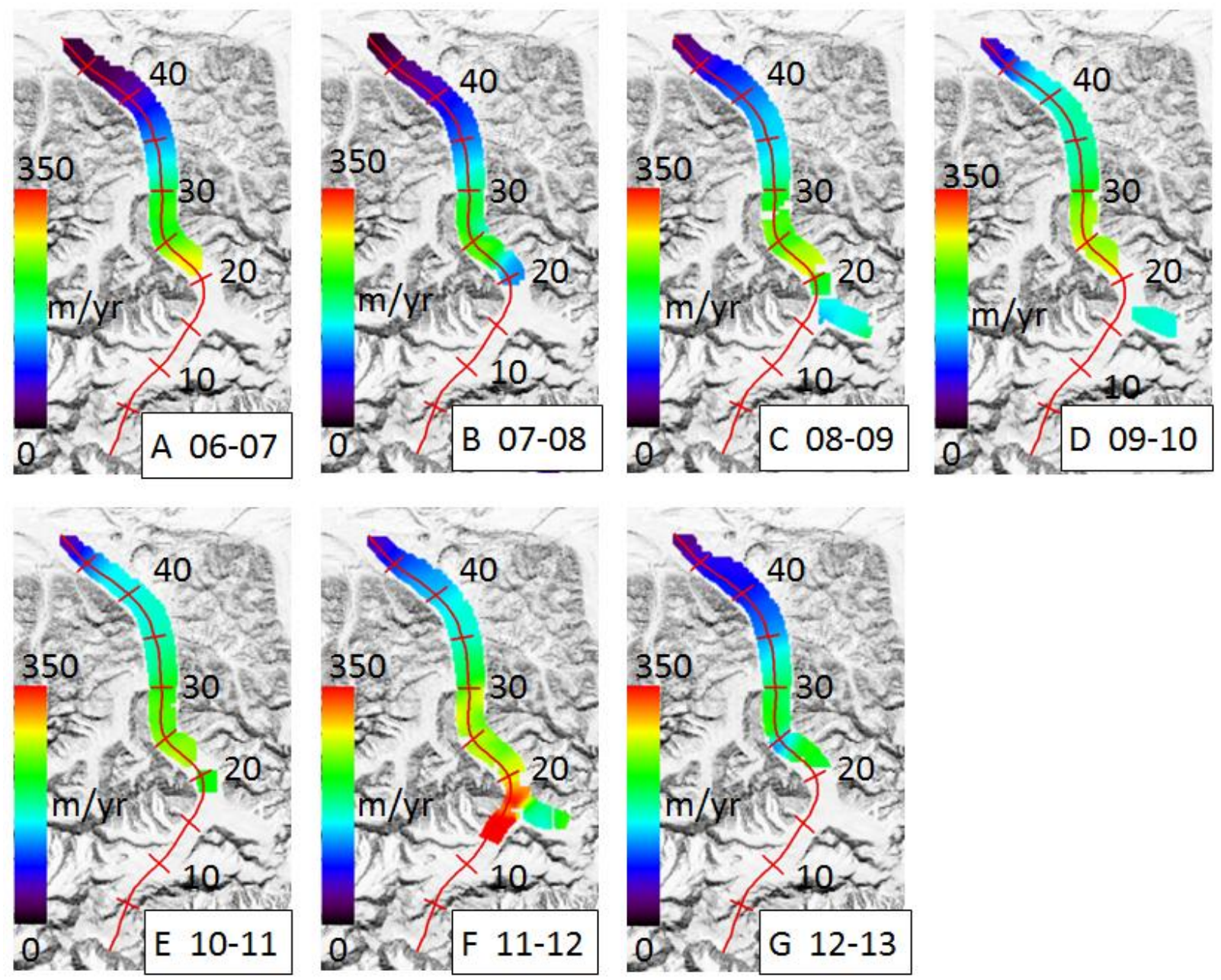

Figure S14. Velocity fields for Matanuska Glacier encompassing the second pulse from 200607 to 2012-13. A) 2006-07 velocity. B) 2007-08 velocity. C) 2008-09 velocity. D) 2009-10 velocity. E) 2010-11 velocity. F) 2011-12 velocity. G) 2012-13 velocity. Velocity fields for the first pulse of Matanuska Glacier are not shown. 

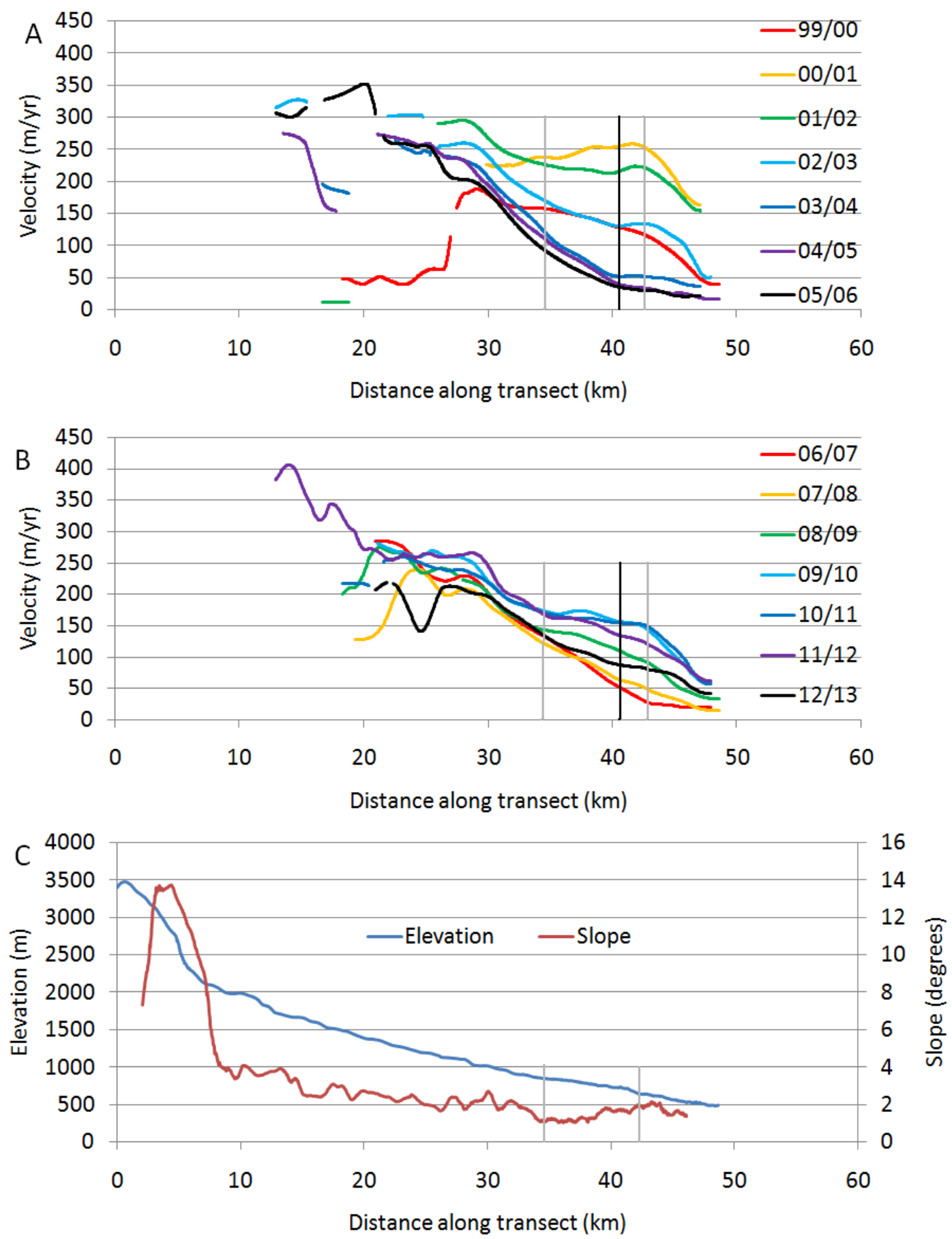

Figure S15. Centerline profiles for Matanuska Glacier. A) Velocity profiles from 1999-00 to 2005-06 showing the first pulse. B) Velocity profiles from 2006-07 to 2012-13 showing the second pulse. C). Elevation and slope of the glacier surface. Vertical black line at $41 \mathrm{~km}$ in panels $\mathrm{A}$ and $\mathrm{B}$ indicates position at which velocity values were taken to create time-series in Fig. S16. Vertical gray lines at 34 and $43 \mathrm{~km}$ indicate locations of major change in bedrock lithology. 


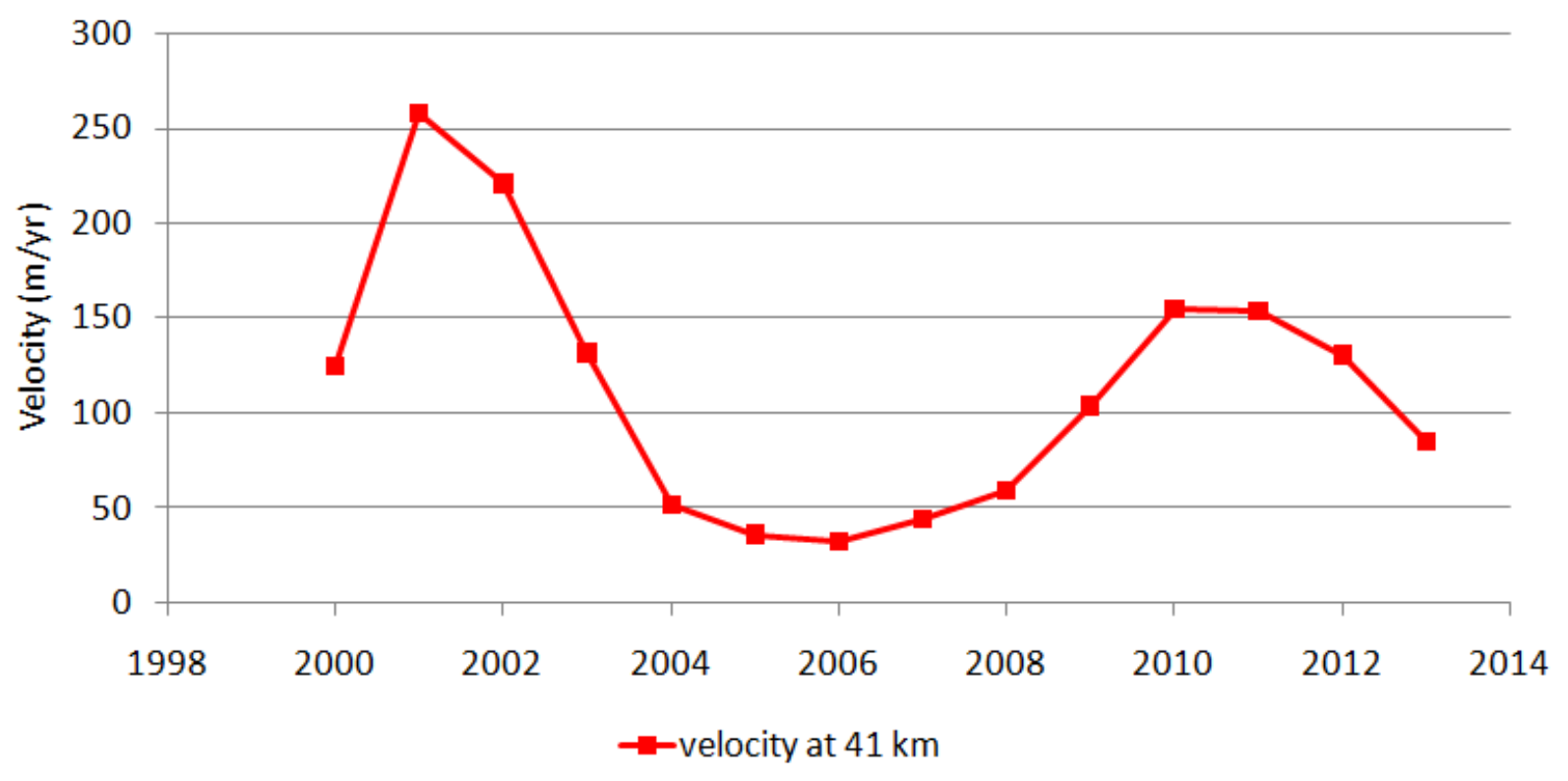

Figure S16. Velocity of Matanuska Glacier through time, at $41 \mathrm{~km}$. Two pulses are seen; the first pulse peaked in 2001 at $257.5 \mathrm{~m} / \mathrm{yr}$, and the second pulse peaked in 2010 at $154.5 \mathrm{~m} / \mathrm{yr}$. 


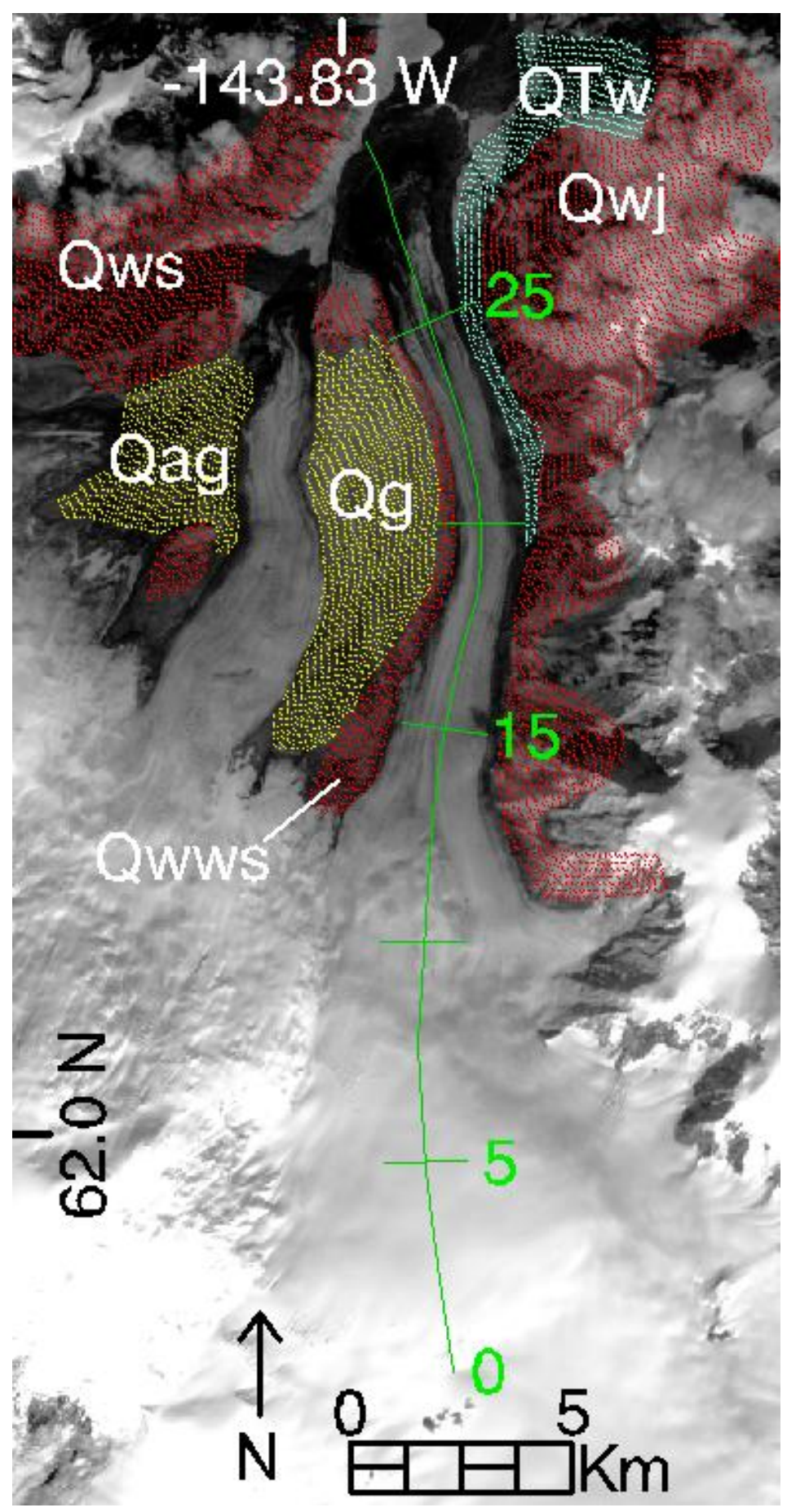

Figure S17. Copper Glacier. Transect along glacier centerline (shown in green) indicates the location of the velocity profiles shown in Figure S19, units are km. Areas highlighted in aqua (QTw) or red (Qwj, Qwws) indicate areas underlain by crystalline bedrock composed of andesitic lava flows. Areas highlighted in yellow (Qag, Qg) indicate areas underlain by unlithified sediments of Holocene or Pleistocene age, respectively (Richter et al., 2006). 


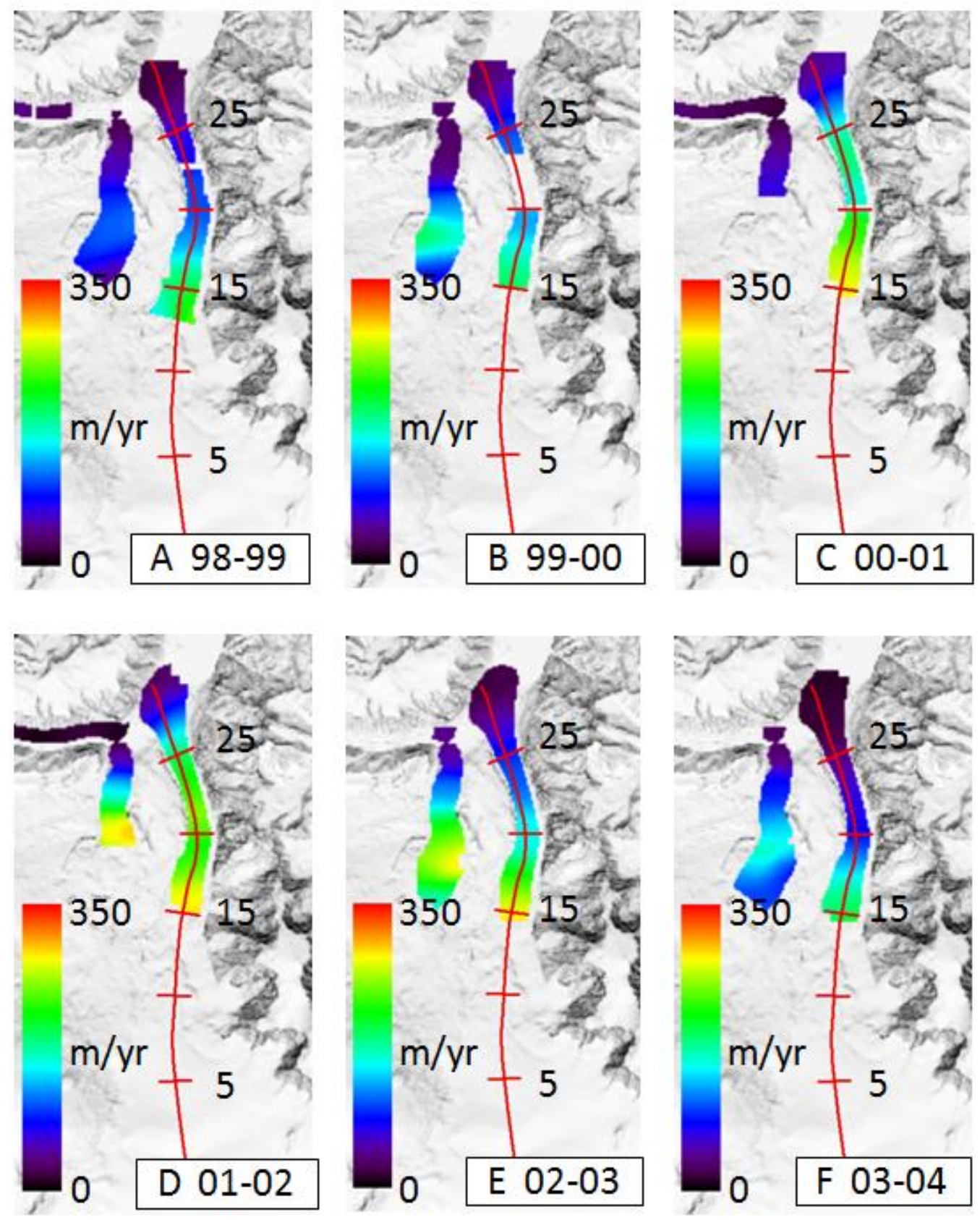

Figure S18. Velocity fields for Copper Glacier encompassing its pulse from 1998-99 to 200304. A) 1998-99 velocity. B) 1999-00 velocity. C) 2000-01 velocity. D) 2001-02 velocity. E) 2002-03 velocity. F) 2003-04 velocity. Velocity fields from 2004-05 to 2012-13 are not shown. 

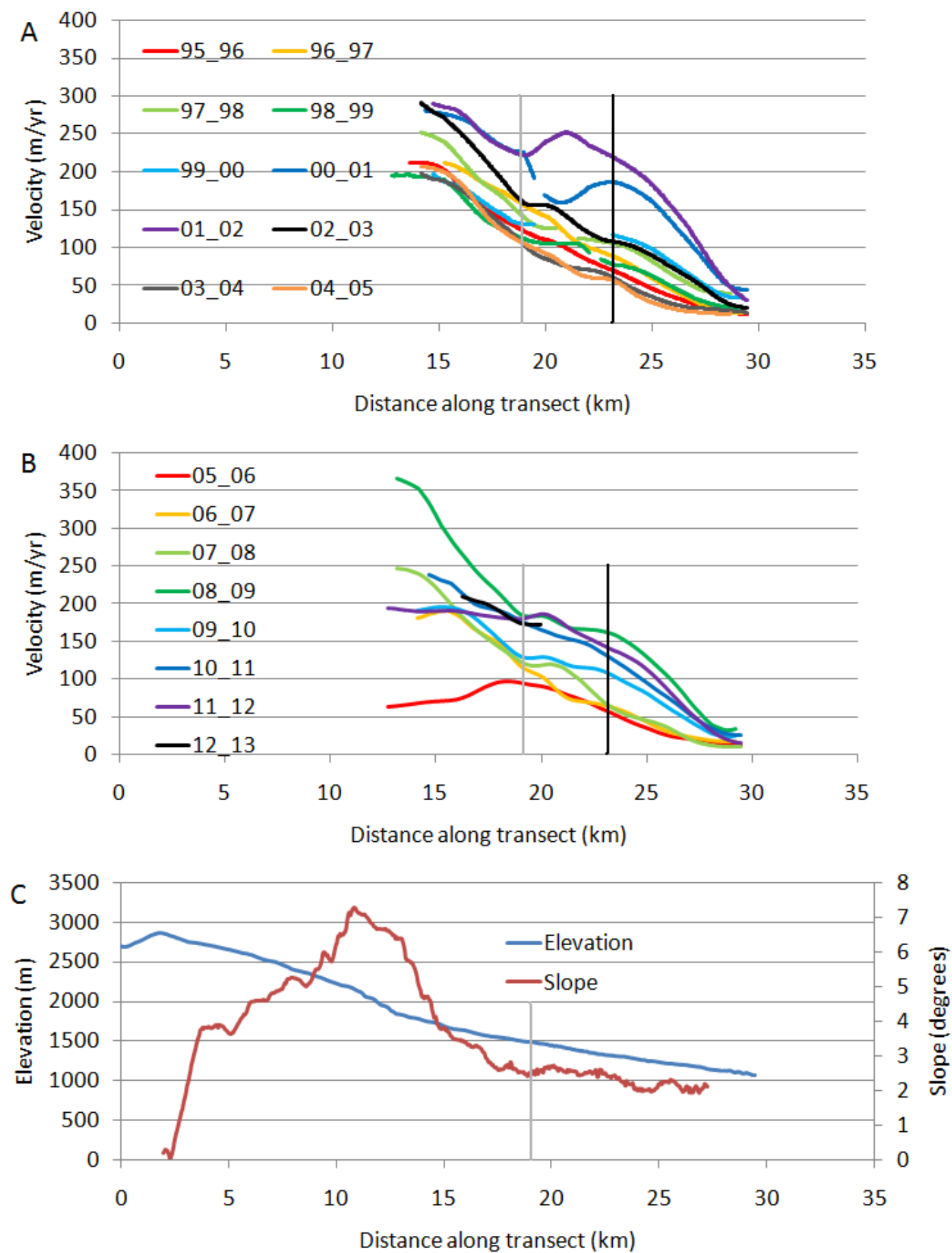

Figure S19. Centerline profiles for Copper Glacier. A) Velocity profiles from 1995-96 to 200405 showing the first pulse. B) Velocity profiles from 2005-06 to 2012-13 showing the acceleration phase of the second pulse. C). Elevation and slope of the glacier surface. Vertical black line at $23 \mathrm{~km}$ in panels $\mathrm{A}$ and $\mathrm{B}$ indicates position at which velocity values were taken to create time-series in Fig. S20. Vertical gray line at $19 \mathrm{~km}$ indicates location of major change in bedrock lithology. 


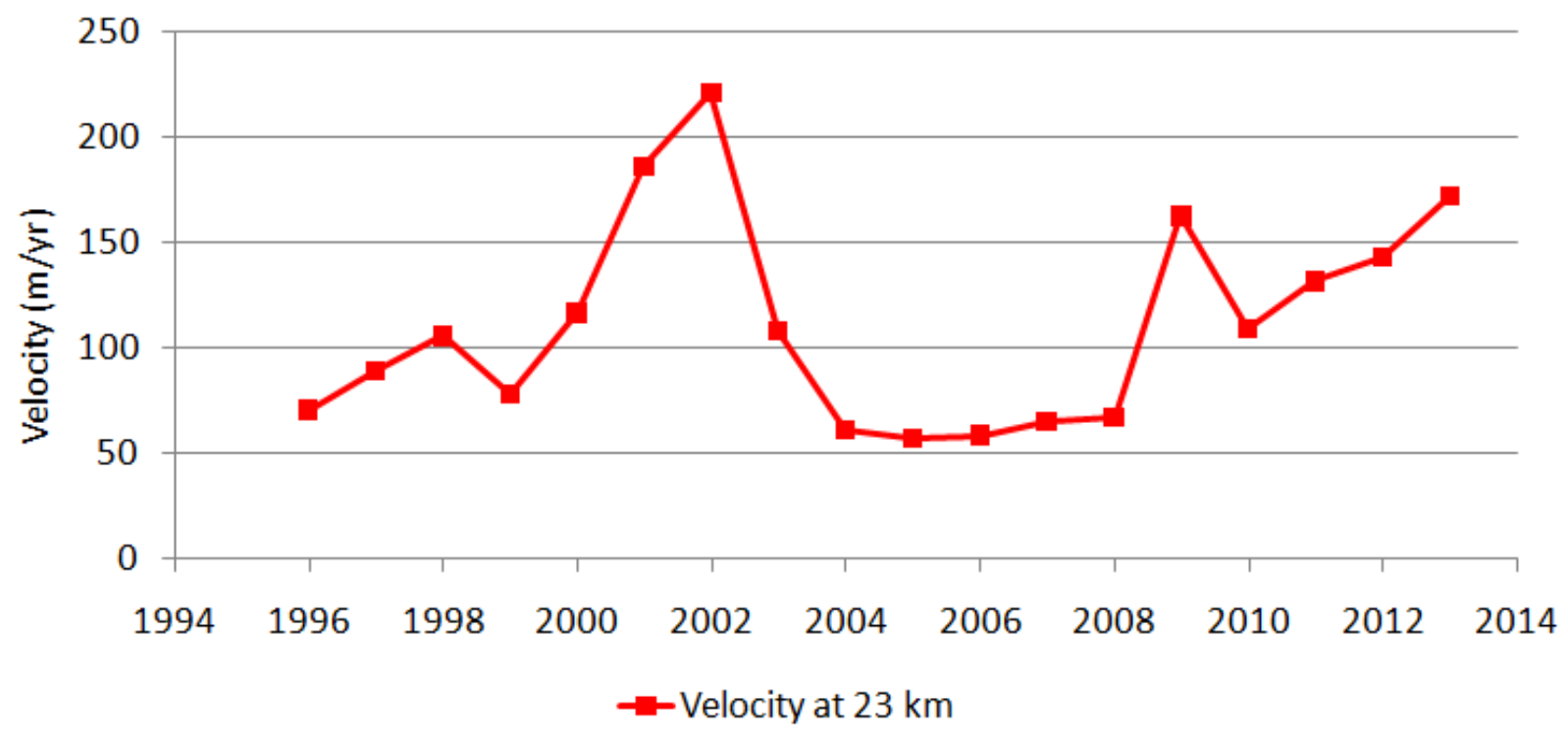

Figure S20. Velocity of Copper Glacier through time, at $23 \mathrm{~km}$. One complete pulse cycle is seen, with a peak in 2002 of $220.2 \mathrm{~m} / \mathrm{yr}$, and the acceleration phase of a second pulse is seen, with its current peak in 2013 of $172.0 \mathrm{~m} / \mathrm{yr}$. 


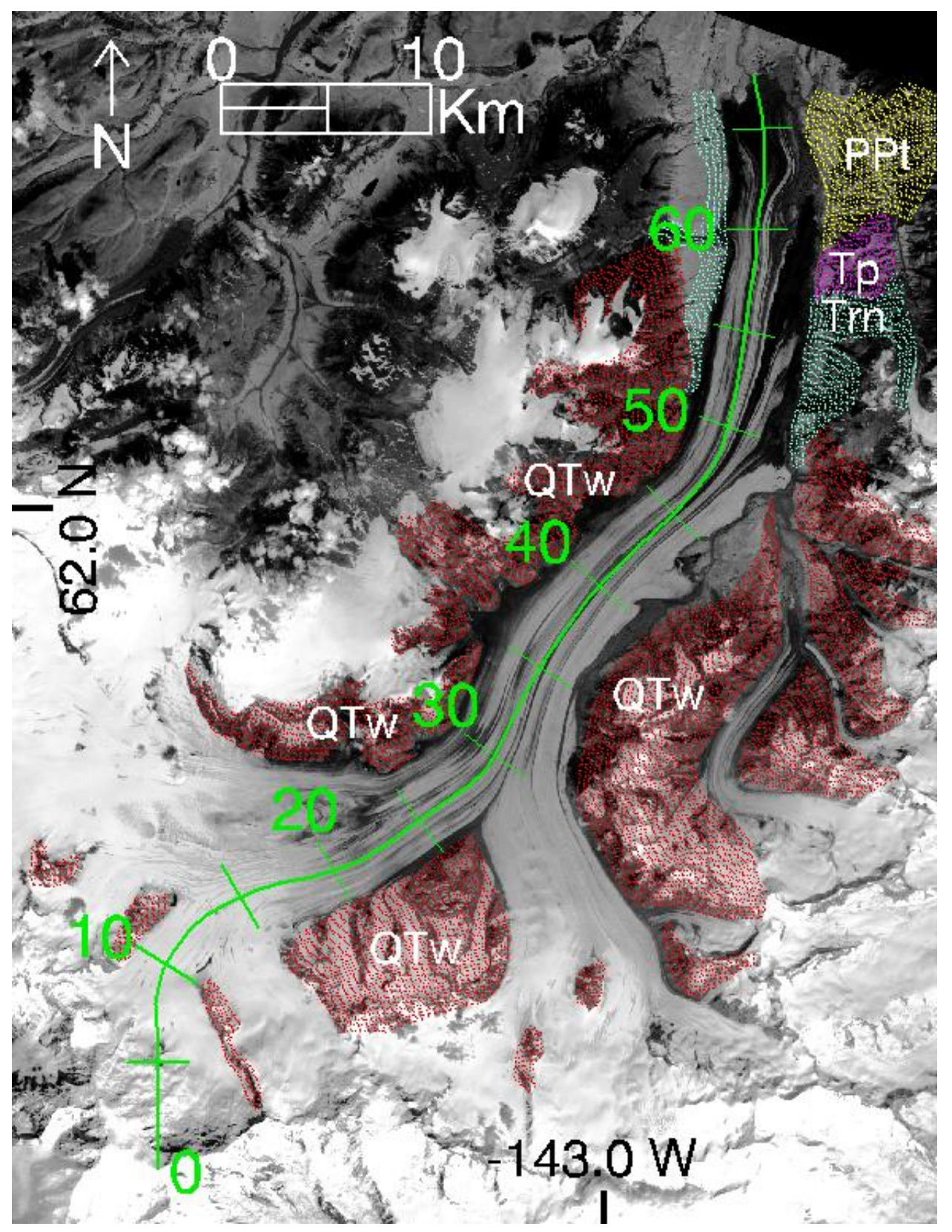

Figure S21. Nabesna Glacier. Transect along glacier centerline (shown in green) indicates the location of the velocity profiles shown in Figure S23, units are $\mathrm{km}$. Areas highlighted in red (QTw), aqua (Trn), or yellow (PPt) indicate areas underlain by crystalline bedrock composed of lava. Areas highlighted by magenta (Tp) indicate areas underlain by granitic bedrock (Richter et al., 2006). 

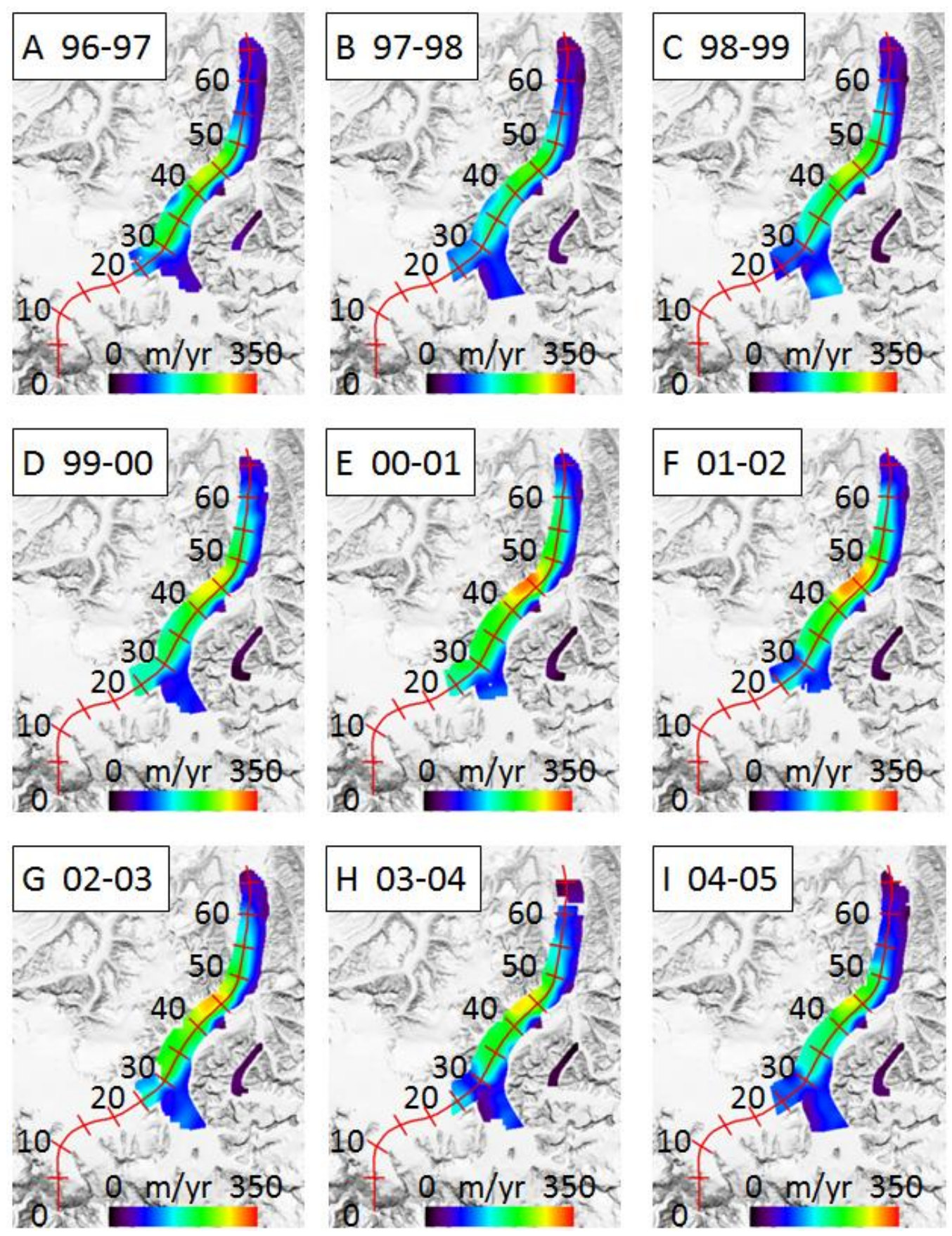

Figure S22. Velocity fields for Nabesna Glacier encompassing its pulse from 1996-97 to 200405. A) 1996-97 velocity. B) 1997-98 velocity. C) 1998-99 velocity. D) 1999-00 velocity. E) 2000-01 velocity. F) 2001-02 velocity. G) 2002-03 velocity. H) 2003-04 velocity. I) 2004-05 velocity. Velocity fields from $2005-06$ to $2012-13$ are not shown. 

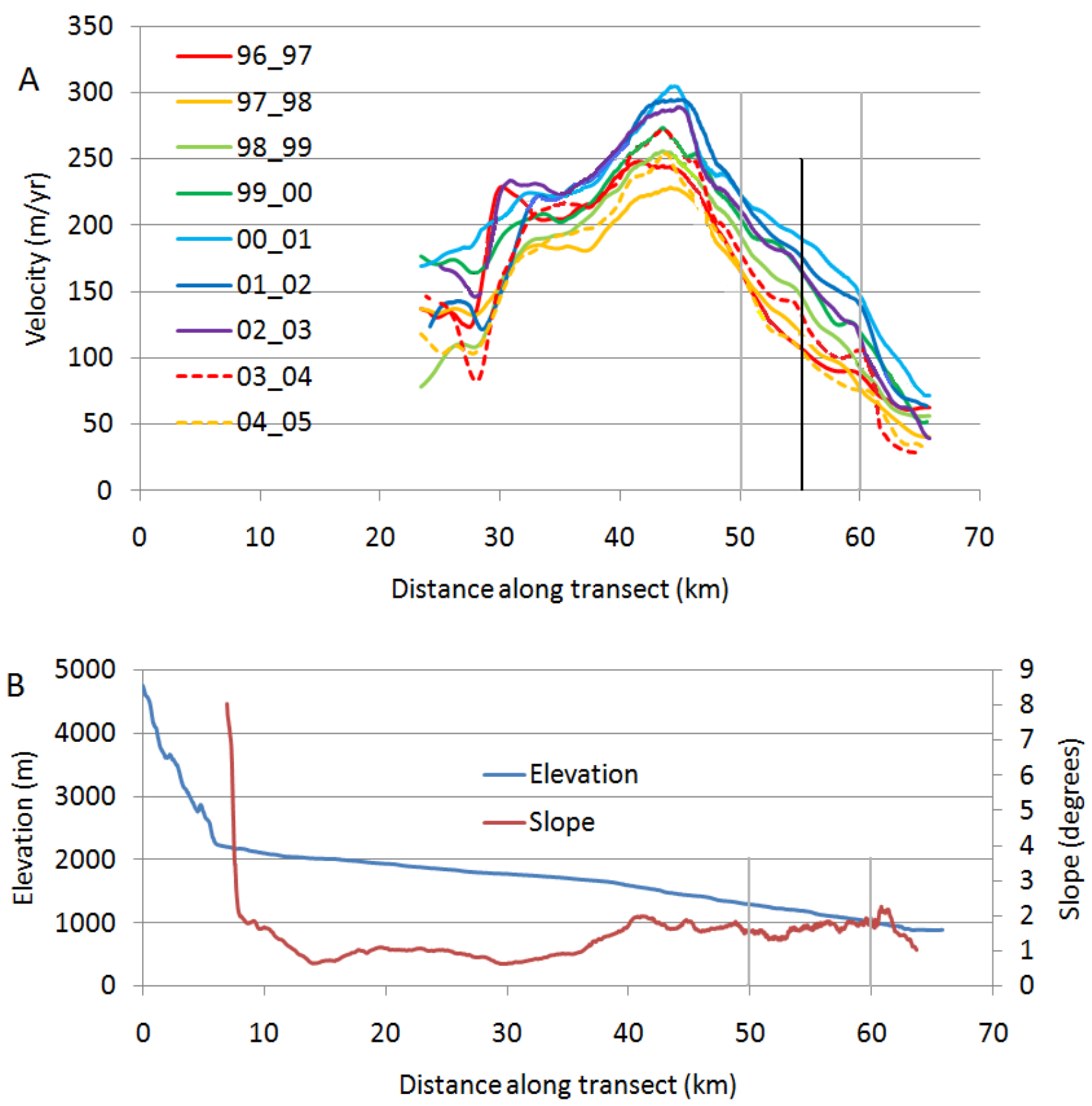

Figure S23. Centerline profiles for Nabesna Glacier. A) Velocity profiles from 1996-97 to 2004-05 showing the pulse. Dashed black profile represents a composite of velocity measurements by Li et al. (2008) spanning Jan., 1994 to May, 1996. B). Elevation and slope of the glacier surface. Vertical black line at $55 \mathrm{~km}$ in panel A indicates position at which velocity values were taken to create time-series in Fig. S24. Vertical gray lines at 50 and $60 \mathrm{~km}$ indicate locations of major change in bedrock lithology. 


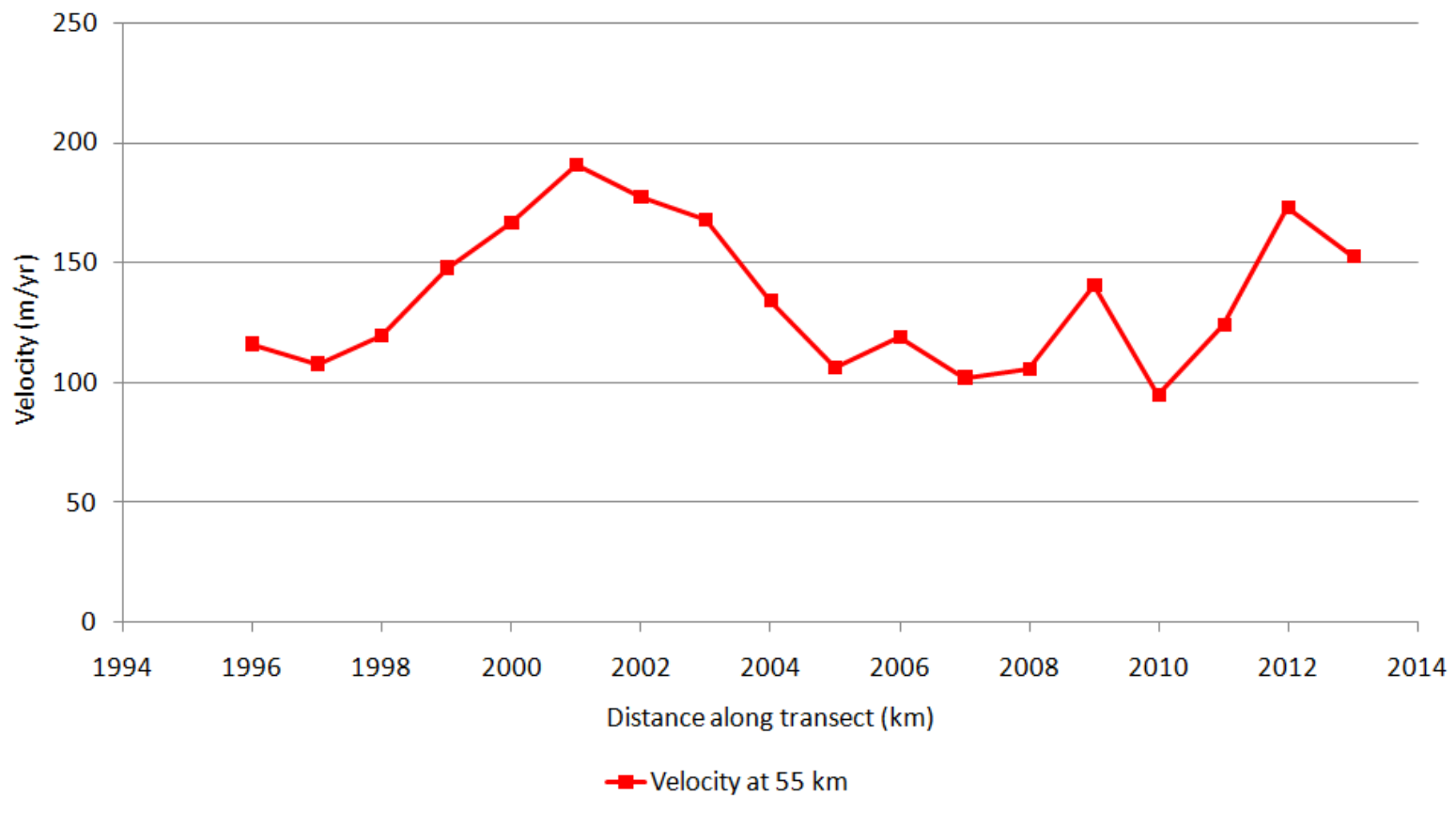

Figure S24. Velocity of Nabesna Glacier through time, at $55 \mathrm{~km}$. One complete pulse cycle is seen, with a peak in 2001 of $190.6 \mathrm{~m} / \mathrm{yr}$. 


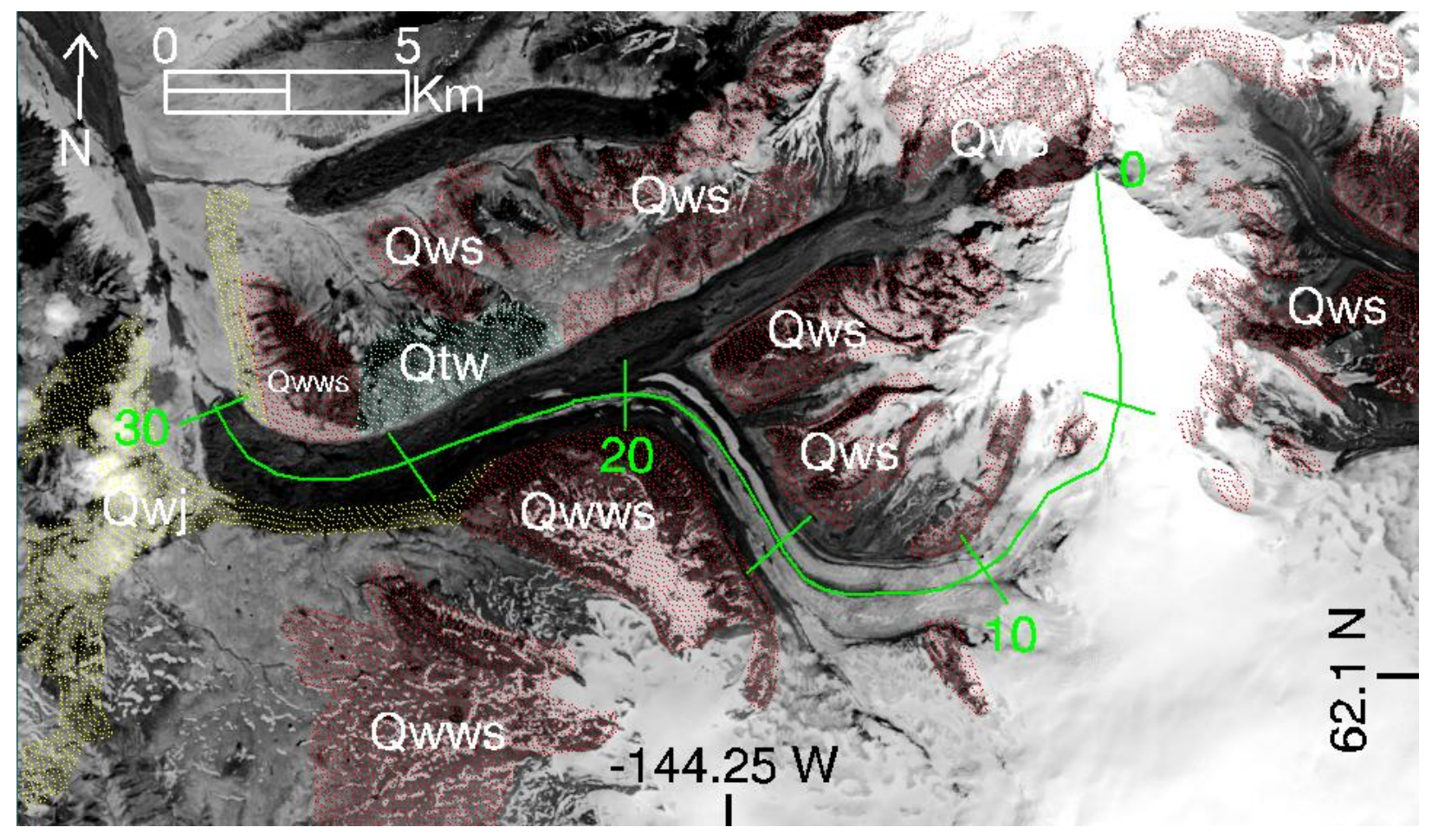

Figure S25. Sanford Glacier. Transect along glacier centerline (shown in green) indicates the location of the velocity profiles shown in Figure S27, units are km. Areas highlighted in red (Qws, Qwws), aqua (Qtw), or yellow (Qwj) indicate areas underlain by crystalline bedrock composed of lava (Richter et al., 2006). 

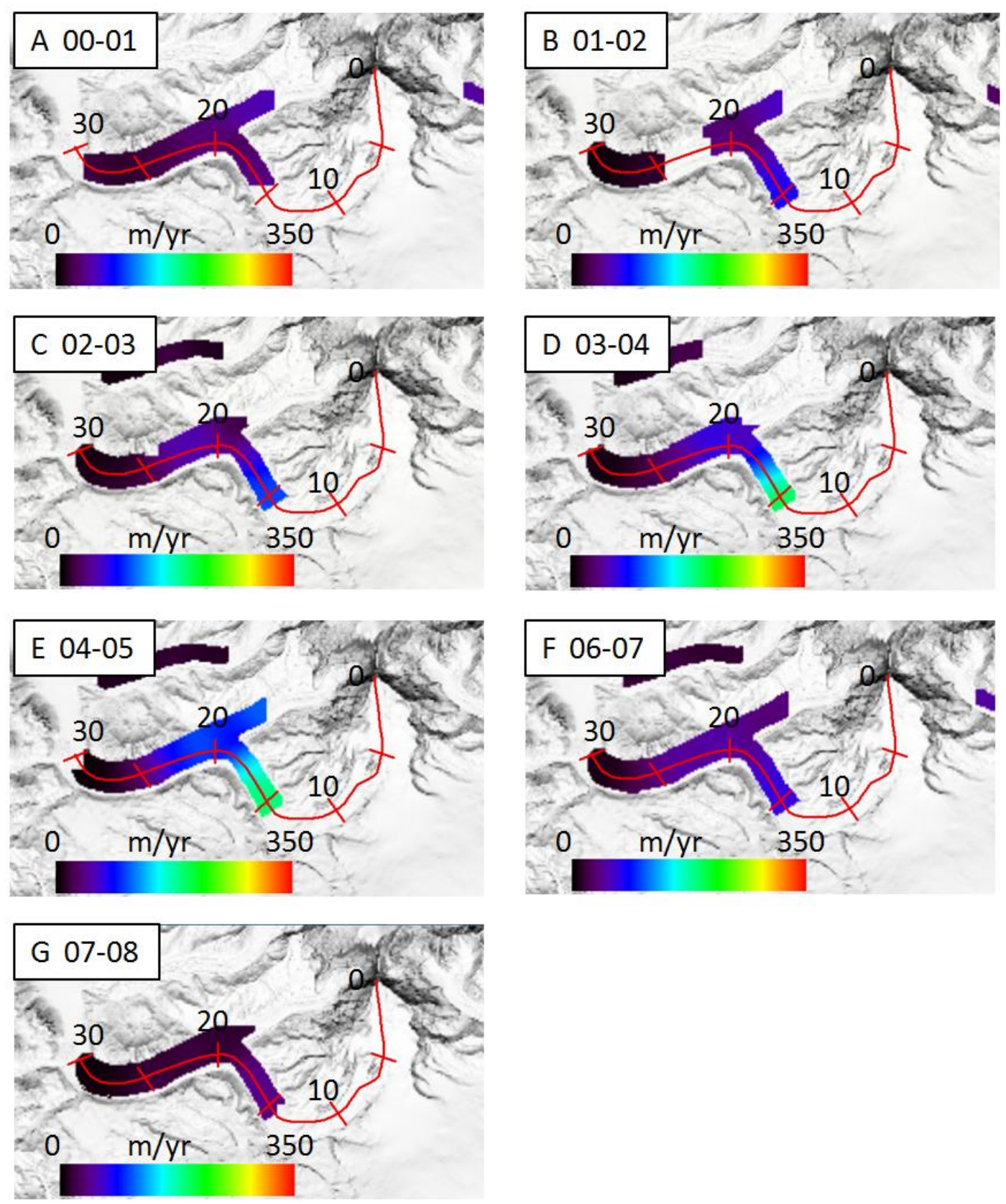

Figure S26. Velocity fields for Sanford Glacier encompassing its pulse from 2000-01 to 200708. A) 2000-01 velocity. B) 2001-02 velocity. C) 2002-03 velocity. D) 2003-04 velocity. E) 2004-05 velocity. F) 2006-07 velocity. G) 2007-08 velocity. 

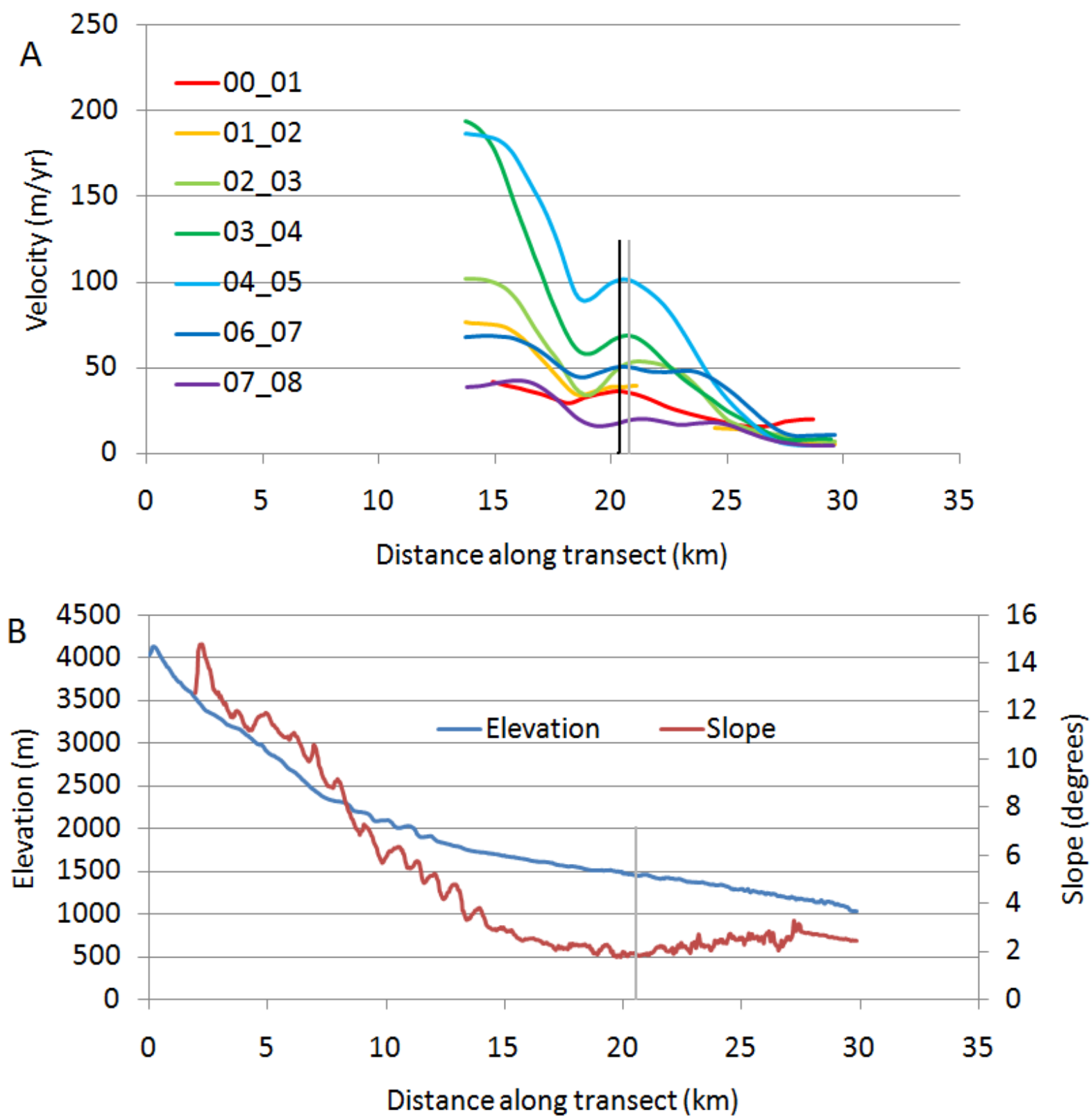

Figure S27. Centerline profiles for Sanford Glacier. A) Velocity profiles from 2000-01 to 200708 showing the pulse. B). Elevation and slope of the glacier surface. Vertical black line at 20.5 $\mathrm{km}$ in panel A indicates position at which velocity values were taken to create time-series in Fig. S28. Vertical gray line at $21 \mathrm{~km}$ indicates location of major change in bedrock lithology. 


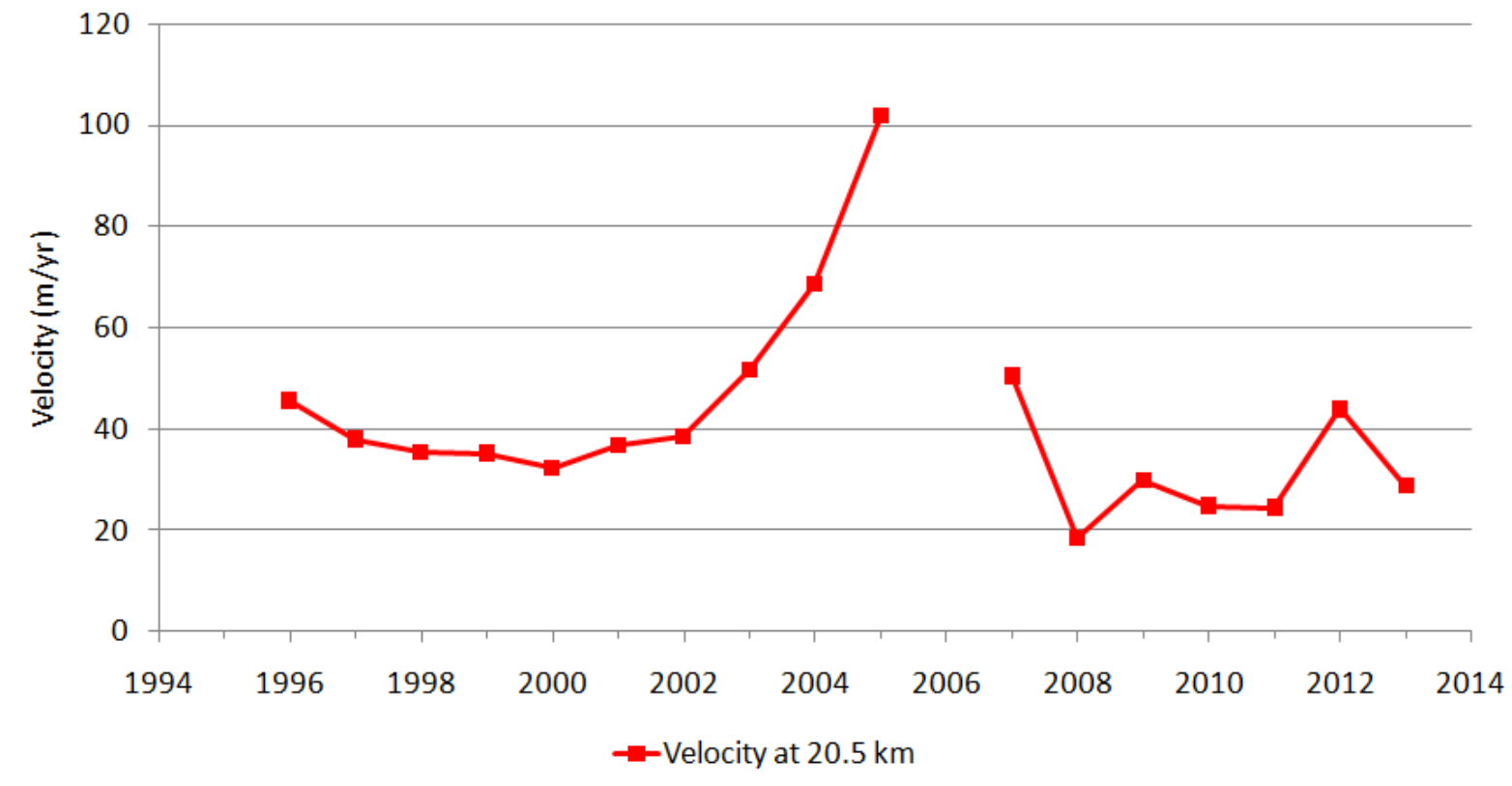

Figure S28. Velocity of Sanford Glacier through time, at $20.5 \mathrm{~km}$. One pulse cycle is seen, with a peak in 2005 of $101.7 \mathrm{~m} / \mathrm{yr}$. 
Table S1. Landsat image pairs used to produce velocity fields for the Central Alaska Range.

\begin{tabular}{|c|c|c|c|c|c|c|c|}
\hline \multicolumn{2}{|c|}{ Path/Row } & \multirow{2}{*}{$\frac{\text { Date } 1}{24-\text { Sep-73 }}$} & Sensor & \multicolumn{2}{|c|}{ Path/Row } & \multirow{2}{*}{$\begin{array}{c}\text { Date } 2 \\
27-\text { Jul-74 }\end{array}$} & Sensor \\
\hline WRS 1 & $76 / 16$ & & MSS 1 & WRS 1 & $76 / 16$ & & MSS 1 \\
\hline WRS 1 & $76 / 16$ & $27-J u l-74$ & MSS 1 & WRS 1 & $76 / 16$ & $23-\mathrm{Sep}-75$ & MSS 2 \\
\hline WRS 1 & $76 / 16$ & $23-$ Sep- 75 & MSS 2 & WRS 1 & $76 / 16$ & 7-Jul-76 & MSS 2 \\
\hline WRS 1 & $76 / 16$ & 7-Jul-76 & MSS 2 & WRS 1 & $76 / 16$ & $1-J u l-77$ & MSS 2 \\
\hline WRS 1 & $76 / 16$ & $1-J u l-77$ & MSS 2 & WRS 1 & $76 / 16$ & $2-\operatorname{Aug}-78$ & MSS 2 \\
\hline WRS 1 & $76 / 16$ & 2-Aug-78 & MSS 2 & WRS 1 & $76 / 16$ & 22-Jul-80 & MSS 2 \\
\hline WRS 1 & $76 / 16$ & $22-J u l-80$ & MSS 2 & WRS 1 & $76 / 16$ & $4-A u g-81$ & MSS 2 \\
\hline WRS 1 & $76 / 16$ & $4-A u g-81$ & MSS 2 & WRS 1 & $76 / 16$ & 3-Jul-82 & MSS 3 \\
\hline WRS 1 & $76 / 16$ & 3-Jul-82 & MSS 3 & WRS2 & $70 / 16$ & 18-Aug-83 & MSS 4 \\
\hline WRS2 & $70 / 16$ & $18-$ Aug -83 & MSS 4 & WRS2 & $70 / 16$ & $28-\operatorname{Aug}-84$ & MSS 5 \\
\hline WRS 2 & $70 / 16$ & $28-\operatorname{Aug}-84$ & MSS 5 & WRS 2 & $70 / 16$ & $16-\mathrm{Sep}-85$ & MSS 5 \\
\hline WRS2 & $70 / 16$ & $16-\mathrm{Sep}-85$ & TM 5 & WRS2 & $70 / 16$ & $17-J u l-86$ & TM 5 \\
\hline WRS2 & $70 / 16$ & $17-J u l-86$ & TM 5 & WRS2 & $70 / 16$ & 21-Aug-87 & TM 5 \\
\hline WRS 2 & $70 / 16$ & $21-$ Aug-87 & TM 5 & WRS2 & $70 / 16$ & 29-Jun-91 & TM 5 \\
\hline WRS2 & $70 / 16$ & 29-Jun-91 & TM 5 & WRS2 & $70 / 16$ & 26-Aug-92 & TM 4 \\
\hline WRS2 & $70 / 16$ & $26-$ Aug- 92 & TM 4 & WRS2 & $70 / 16$ & $9-S e p-94$ & TM 5 \\
\hline WRS2 & $70 / 16$ & $9-\mathrm{Sep}-94$ & TM 5 & WRS2 & $70 / 16$ & 8-Jun-95 & TM 5 \\
\hline WRS2 & $70 / 16$ & 8-Jun-95 & TM 5 & WRS2 & $70 / 16$ & 19-Jun-99 & TM 5 \\
\hline WRS2 & $70 / 16$ & 19-Jun-99 & TM 5 & WRS2 & $70 / 16$ & $16-$ Aug-00 & ETM+7 \\
\hline WRS2 & $70 / 16$ & $10-A p r-00$ & ETM+7 & WRS2 & $70 / 16$ & 29-Apr-01 & ETM+7 \\
\hline WRS 2 & $70 / 16$ & 29-Apr-01 & ETM+7 & WRS2 & $70 / 16$ & $16-\operatorname{Apr}-02$ & ETM+7 \\
\hline WRS2 & $70 / 16$ & $16-A p r-02$ & ETM+7 & WRS2 & $70 / 16$ & 3-Apr-03 & ETM+7 \\
\hline WRS 2 & $70 / 16$ & 9-Aug-03 & ETM+7 & WRS2 & $70 / 16$ & $10-J u l-04$ & ETM+7 \\
\hline WRS2 & $70 / 16$ & $10-J u l-04$ & ETM+7 & WRS2 & $70 / 16$ & $14-$ Aug-05 & ETM+7 \\
\hline WRS2 & $70 / 16$ & 14 -Aug-05 & ETM+7 & WRS2 & $70 / 16$ & $10-\mathrm{Sep}-06$ & TM 5 \\
\hline WRS2 & $70 / 16$ & $10-\mathrm{Sep}-06$ & TM 5 & WRS2 & $70 / 16$ & 28-Aug-07 & TM 5 \\
\hline WRS 2 & $70 / 16$ & 28-Aug-07 & TM 5 & WRS2 & $70 / 16$ & $5-J u l-08$ & ETM+7 \\
\hline WRS2 & $70 / 16$ & 5-Jul-08 & ETM+7 & WRS2 & $70 / 16$ & 8-Jul-09 & ETM+7 \\
\hline WRS2 & $70 / 16$ & 8-Jul-09 & ETM+7 & WRS2 & $70 / 16$ & $20-$ Aug-10 & TM 5 \\
\hline WRS 2 & $70 / 16$ & $20-$ Aug-10 & TM 5 & WRS2 & $70 / 16$ & $6-J u l-11$ & TM 5 \\
\hline WRS2 & $70 / 16$ & $18-0 c t-11$ & ETM+7 & WRS2 & $70 / 16$ & $20-0 c t-12$ & ETM+7 \\
\hline
\end{tabular}


Table S2. Landsat image pairs used to produce velocity fields for the West Alaska Range.

\begin{tabular}{|c|c|c|c|c|c|c|c|}
\hline Path & /Row & Date 1 & Sensor & Patl & Row & Date 2 & Sensor \\
\hline WRS 1 & $76 / 17$ & 24-Sep-73 & MSS 1 & WRS1 & $76 / 17$ & 14-Aug-74 & MSS 1 \\
\hline WRS 1 & $76 / 17$ & 14-Aug-74 & MSS 1 & WRS1 & $76 / 17$ & 23-Sep-75 & MSS 2 \\
\hline WRS 1 & $76 / 17$ & 23-Sep-75 & MSS 2 & WRS1 & $76 / 17$ & 30-Aug-76 & MSS 2 \\
\hline WRS1 & $76 / 17$ & $30-$ Aug- 76 & MSS 2 & WRS1 & $76 / 17$ & $2-$ Aug -78 & MSS 2 \\
\hline WRS 1 & $76 / 17$ & 2-Aug-78 & MSS 2 & WRS1 & $76 / 17$ & 24-Aug-79 & MSS 3 \\
\hline WRS 1 & $76 / 17$ & $24-$ Aug-79 & MSS 3 & WRS1 & $76 / 17$ & 22-Jul-80 & MSS 2 \\
\hline WRS1 & $76 / 17$ & 22-Jul-80 & MSS 2 & WRS1 & $76 / 17$ & 4-Aug-81 & MSS 2 \\
\hline WRS 1 & $76 / 17$ & 6-May-81 & MSS 2 & WRS1 & $76 / 17$ & $4-A p r-82$ & MSS 3 \\
\hline WRS 1 & $76 / 17$ & 3-Jul-82 & MSS 3 & WRS2 & $70 / 17$ & $2-\operatorname{Aug}-83$ & MSS 4 \\
\hline WRS2 & $70 / 17$ & $2-A u g-83$ & MSS 4 & WRS2 & $70 / 17$ & 12-Aug-84 & MSS 5 \\
\hline WRS2 & $70 / 17$ & $12-\mathrm{Aug}-84$ & MSS 5 & WRS2 & $70 / 17$ & 16-Sep-85 & MSS 5 \\
\hline WRS 2 & $70 / 17$ & $16-\mathrm{Sep}-85$ & TM 5 & WRS2 & $70 / 17$ & 17-Jul-86 & TM 5 \\
\hline WRS2 & $70 / 17$ & 17-Jul-86 & TM 5 & WRS2 & $70 / 17$ & 21-Aug-87 & TM 5 \\
\hline WRS2 & $70 / 17$ & $15-$ Sep-99 & ETM+7 & WRS2 & $70 / 17$ & 16-Aug-00 & $\mathrm{ETM}+7$ \\
\hline WRS 2 & $70 / 17$ & 12-May-00 & ETM+7 & WRS2 & $70 / 17$ & 29-Apr-01 & ETM+7 \\
\hline WRS2 & $70 / 17$ & $16-J u n-01$ & ETM+7 & WRS2 & $70 / 17$ & 18-May-02 & $\mathrm{ETM}+7$ \\
\hline WRS2 & $70 / 17$ & 5-Jul-02 & ETM+7 & WRS2 & $70 / 17$ & 9-Aug-03 & $\mathrm{ETM}+7$ \\
\hline WRS 2 & $70 / 17$ & 9-Aug-03 & ETM+7 & WRS2 & $70 / 17$ & 10-Jul-04 & ETM+7 \\
\hline WRS 2 & $70 / 17$ & 10-Jul-04 & ETM+7 & WRS2 & $70 / 17$ & 14-Aug-05 & ETM+7 \\
\hline WRS2 & $70 / 17$ & $24-A p r-05$ & ETM+7 & WRS2 & $70 / 17$ & 27-Apr-06 & $\mathrm{ETM}+7$ \\
\hline WRS2 & $70 / 17$ & 27-Apr-06 & ETM+7 & WRS2 & $70 / 17$ & 30-Apr-07 & ETM+7 \\
\hline WRS2 & $70 / 17$ & $28-\mathrm{Aug}-07$ & TM 5 & WRS2 & $70 / 17$ & 6-Aug-08 & $\mathrm{ETM}+7$ \\
\hline WRS2 & $70 / 17$ & 6-Aug-08 & ETM+7 & WRS2 & $70 / 17$ & 30-Jun-09 & TM 5 \\
\hline WRS2 & $70 / 17$ & 30-Jun-09 & TM 5 & WRS2 & $70 / 17$ & 20-Aug-10 & TM 5 \\
\hline WRS 2 & $70 / 17$ & 8-May-10 & ETM+7 & WRS2 & $70 / 17$ & $9-A p r-11$ & $\mathrm{ETM}+7$ \\
\hline WRS 2 & $70 / 17$ & $20-$ Aug-10 & TM 5 & WRS2 & $70 / 17$ & 15-Aug-11 & ETM+7 \\
\hline WRS2 & $70 / 17$ & 9-Apr-11 & ETM+7 & WRS2 & $70 / 17$ & 27-Apr-12 & $\mathrm{ETM}+7$ \\
\hline WRS2 & $70 / 17$ & 27-Apr-12 & ETM+7 & WRS2 & $70 / 17$ & 14-Apr-13 & ETM+7 \\
\hline
\end{tabular}


Table S3. Landsat image pairs used to make velocity fields for the Chugach Mountains.

\begin{tabular}{|c|c|c|c|c|c|c|c|}
\hline \multicolumn{2}{|c|}{ Path/Row } & Date 1 & Sensor & \multicolumn{2}{|c|}{ Path/Row } & Date 2 & Sensor \\
\hline WRS1 & $73 / 17$ & $8-\operatorname{sep}-72$ & MSS 1 & WRS1 & $73 / 17$ & 16-Aug-73 & MSS 1 \\
\hline WRS 1 & $73 / 17$ & $16-$ Aug -73 & MSS 1 & WRS 1 & $73 / 17$ & 6-Jul-74 & MSS 1 \\
\hline WRS 1 & $73 / 17$ & $25-A p r-74$ & MSS 1 & WRS 1 & $73 / 17$ & $11-$ Apr -75 & MSS 2 \\
\hline WRS 1 & $73 / 17$ & $10-\mathrm{Jul}-75$ & MSS 2 & WRS 1 & $73 / 17$ & $2-0 c t-76$ & MSS 2 \\
\hline WRS 1 & $73 / 17$ & $2-0 c t-76$ & MSS 2 & WRS 1 & $73 / 17$ & $22-$ Aug- 77 & MSS 2 \\
\hline WRS 1 & $73 / 17$ & 22-Aug-77 & MSS 2 & WRS 1 & $73 / 17$ & $26-$ Aug- 78 & MSS 3 \\
\hline WRS 1 & $73 / 17$ & $26-$ Aug-78 & MSS 3 & WRS 1 & $73 / 17$ & $8-S e p-79$ & MSS 3 \\
\hline WRS1 & $73 / 17$ & $8-\mathrm{Sep}-79$ & MSS 3 & WRS 1 & $73 / 17$ & $24-$ Aug-80 & MSS 2 \\
\hline WRS 1 & $73 / 17$ & $24-\operatorname{Aug}-80$ & MSS 2 & WRS 1 & $73 / 17$ & 21-May-81 & MSS 2 \\
\hline WRS 1 & $73 / 17$ & $21-$ May-81 & MSS 2 & WRS 1 & $73 / 17$ & 25-May-82 & MSS 3 \\
\hline WRS 1 & $73 / 17$ & 23-Aug-82 & MSS 3 & WRS2 & $67 / 17$ & $28-J u l-83$ & MSS 4 \\
\hline WRS 1 & $70 / 17$ & $28-\mathrm{Jul}-83$ & MSS 4 & WRS 1 & $70 / 17$ & 20-Jun-84 & MSS 5 \\
\hline WRS 1 & $70 / 17$ & $20-J u n-84$ & MSS 5 & WRS 1 & $70 / 17$ & $11-\mathrm{sep}-85$ & MSS 5 \\
\hline WRS2 & $67 / 17$ & $11-\mathrm{Sep}-85$ & MSS 5 & WRS 2 & $67 / 17$ & 28-Jul-86 & TM 5 \\
\hline WRS2 & $67 / 17$ & $28-J u l-86$ & TM 5 & WRS2 & $67 / 17$ & 15-Jul-87 & TM 5 \\
\hline WRS2 & $67 / 17$ & $15-J u l-87$ & TM 5 & WRS2 & $67 / 17$ & 12-Jul-89 & TM 4 \\
\hline WRS2 & $67 / 17$ & $10-$ Sep-99 & ETM+7 & WRS2 & $67 / 17$ & $11-$ Aug-00 & ETM+7 \\
\hline WRS2 & $67 / 17$ & $11-$ Aug-00 & ETM+7 & WRS2 & $67 / 17$ & $15-\mathrm{sep}-01$ & ETM+7 \\
\hline WRS2 & $67 / 17$ & 15-Sep-01 & ETM+7 & WRS2 & $67 / 17$ & $1-$ Aug-02 & ETM+7 \\
\hline WRS2 & $67 / 17$ & $1-$ Aug-02 & ETM+7 & WRS2 & $67 / 17$ & 19-Jul-03 & ETM+7 \\
\hline WRS2 & $67 / 17$ & 19-Jul-03 & ETM+7 & WRS2 & $67 / 17$ & 30-Aug-04 & TM 5 \\
\hline WRS2 & $67 / 17$ & $30-$ Aug- 04 & TM 5 & WRS2 & $67 / 17$ & 9-Aug-05 & ETM+7 \\
\hline WRS2 & $67 / 17$ & $9-$ Aug-05 & ETM+7 & WRS2 & $67 / 17$ & $13-\mathrm{sep}-06$ & ETM+7 \\
\hline WRS2 & $67 / 17$ & $13-\mathrm{sep}-06$ & ETM+7 & WRS2 & $67 / 17$ & 28-Jun-07 & ETM+7 \\
\hline WRS2 & $67 / 17$ & $28-J u n-07$ & ETM+7 & WRS2 & $67 / 17$ & $1-$ Aug-0 08 & ETM+7 \\
\hline WRS2 & $67 / 17$ & $1-$ Aug-0 08 & ETM+7 & WRS2 & $67 / 17$ & $5-S e p-09$ & ETM+7 \\
\hline WRS2 & $67 / 17$ & $5-S e p-09$ & ETM+7 & WRS2 & $67 / 17$ & $16-\mathrm{sep}-10$ & TM 5 \\
\hline WRS2 & $67 / 17$ & $16-\mathrm{Sep}-10$ & TM 5 & WRS2 & $67 / 17$ & $11-$ Sep-11 & ETM+7 \\
\hline WRS2 & $67 / 17$ & $11-\mathrm{Sep}-11$ & ETM+7 & WRS2 & $67 / 17$ & 27-Jul-12 & ETM+7 \\
\hline WRS2 & $67 / 17$ & $27-\mathrm{Jul}-12$ & $\mathrm{ETM}+7$ & WRS2 & $67 / 17$ & 30-Jul-13 & ETM+7 \\
\hline
\end{tabular}

